\title{
GALEX UV properties of the polar ring galaxy MCG-05-07-001 and the shell galaxies NGC 1210 and NGC 5329
}

\author{
A. Marino ${ }^{1,5}$, E. Iodice ${ }^{2}$, R. Tantalo ${ }^{3}$, L. Piovan ${ }^{3}$, D. Bettoni ${ }^{1}$, L. M. Buson ${ }^{1}$, C. Chiosi ${ }^{3}$, G. Galletta ${ }^{3}$, \\ R. Rampazzo ${ }^{1}$, and R. M. Rich ${ }^{4}$ \\ 1 INAF - Osservatorio Astronomico di Padova, vicolo dell'Osservatorio 5, 35122 Padova, Italy \\ e-mail: [antonietta.marino; daniela.bettoni;lucio.buson;roberto.rampazzo]@oapd.inaf.it \\ 2 INAF - Osservatorio Astronomico di Capodimonte, via Moiariello 16, 80131 Napoli, Italy \\ e-mail: iodice@na.astro.it \\ 3 Dipartimento di Astronomia Università di Padova, vicolo dell'Osservatorio 2, 35122 Padova, Italy \\ e-mail: [cesare.chiosi;giuseppe.galletta;rosaria.tantalo]@unipd.it, lorenzo.piovan@gmail.com \\ 4 Physics \& Astron. Dept., UCLA, Box 951562, 405 Hilgard Ave., Los Angeles CA 90025-1562, USA \\ e-mail: rmr@astro.ucla.edu \\ 5 Department of Physics and Astronomy, Johns Hopkins University 3400 North Charles Street, Baltimore, MD 21218, USA \\ e-mail: amarino@pha.jhu.edu
}

Received 9 February 2009 / Accepted 19 September 2009

\section{ABSTRACT}

\begin{abstract}
Context. Systems of shells and polar rings in early-type galaxies are considered "bona fide" tracers of mass accretion and/or mergers. Their high frequency in low density environments suggests that these processes could drive the evolution of at least a fraction of the early-type galaxy population.

Aims. We investigate the star formation histories of this type of galaxies. Their UV emission is important for testing whether these galaxies host ongoing or recent star formation and how this formation varies across the galaxy.

Methods. We used far- and near- ultraviolet, optical, near-infrared images, neutral hydrogen HI maps, and line-strength indices to investigate the nuclear and outer regions of these galaxies as well as the regions where fine structures are present.

Results. The GALEX near UV (NUV) and far UV (FUV) images of MCG-05-07-001 and NGC 1210 show complex tidal tails and debris structures. The far UV morphology of both galaxies appears so different from the optical morphology that the early-type classification may not apply. In both GALEX bands, the polar ring of MCG-05-07-001 is the dominant feature, whereas an extended tidal tail dominates the $F U V$ bands of NGC 1210. In MCG-05-07-001 and NGC 1210, there is a strong correlation between structures detected in the FUV and $N U V$ bands and in HI. In contrast, NGC 5329 does not show evidence of shells in the GALEX bands. We try to constrain the age of the accretion episode or merger that produced the shells and polar rings with the aid of composite stellar populations that take the presence of dust into account. The presence of HI in both MCG-05-07-001 and NGC 1210 argues in favour of wet mergers. Models suggest the presence of very young stellar populations in MCG-05-07-001: the observations could be explained in the framework of a conspicuous burst of star formation that occurred $\leq 1 \mathrm{Gyr}$ ago and involved a large fraction of the galaxy mass. Our models suggest that also the nuclei of NGC 1210 and NGC 5329 could have been rejuvenated by an accretion episode about 2-4 Gyr ago.
\end{abstract}

Key words. ultraviolet: galaxies - galaxies: elliptical and lenticular, cD - galaxies: individual: MCG-05-07-001, - galaxies: evolution - galaxies: individual: NGC 1210 - galaxies: NGC 5329

\section{Introduction}

Both early-type galaxies hosting polar rings and those showing fine structure, such as shells, are considered the site of (sometimes major) mass accretion events. In the hierarchical evolutionary scenario they play a special role: namely to fill the gap between ongoing mergers and the quiescent elliptical galaxies.

Polar ring galaxies (PRGs) are peculiar objects consisting of a central spheroidal component, the host galaxy $(H G)$, surrounded by an outer ring $(P R)$, made up of gas, stars, and dust, that the central galaxy (Whitmore et al. 1990). PRGs are quite rare: they amount to only $0.5 \%$ of early-type galaxies in the Local Universe. Being quite difficult to detect, Whitmore et al. (1990) estimated that $4.5 \%$ of the nearby lenticular galaxy population have polar rings. They also suggested that the fraction of

\footnotetext{
* Based on GALEX observations: GI1-059 PI D. Bettoni
}

galaxies that possessed a polar ring at some point in their history could be higher. Indeed, some galaxies may have lost their polar ring because the stability of the polar structure depends also on the impact parameters (Katz \& Rix 1992; Christodoulou et al. 1992). Until some years ago, the most credited formation scenarios for PRGs were either gas accretion by infall or the merger of two gas-rich disk galaxies (Thakar \& Ryden 1996, 1998; Reshetnikov \& Sotnikova 1997; Bekki 1998; Bournaud \& Combes 2003; Bournaud et al. 2005). In the accretion scenario, the accreted material will form a ring that settles into one of the principal planes of the gravitational potential of the host galaxy (Bournaud \& Combes 2003). The merger of two unequal-mass disk galaxies is another way of forming a PRG: the "intruder", on a polar orbit with respect to the "victims" disk, passes close to its center, decelerating and pulling itself back toward the victim by strong dissipation caused by the interaction with the "victim" 
gaseous disk. The morphology of the merger remnants depends on the merging initial orbital parameter and the initial mass ratio of the two galaxies (Bekki 1998; Bournaud \& Combes 2003). Theoretical studies found that an alternative way of producing a PRG is by means of external gas accretion from the cosmic web filaments with inclined angular momentum (Davé et al. 2001; Semelin \& Combes 2005; Macciò et al. 2006). By using highresolution cosmological simulations of galaxy formation in the standard CDM scenario, it has been shown that angular momentum misalignment during hierarchical structure formation can lead to the formation of a high inclined ring/disk (Brook et al. 2008).

Among fine structures, shells are faint, sharp-edged stellar features (Malin \& Carter 1983) characterizing a significant fraction $(\approx 16.5 \%)$ of the field early-type galaxies (Schweizer 1993; Reduzzi et al. 1996; Colbert et al. 2001). Different scenarios for their origin emerge from the rich harvest of simulations performed since their discovery in the early 80's: from major mergers (Barnes 1992; Hernquist \& Spergel 1992; Hernquist \& Mihos 1995), to accretion between galaxies of different masses (mass ratios typically 1/10-1/100) (see e.g. Dupraz \& Combes 1986; Hernquist \& Quinn 1987b,a), and to even weaker interaction events (Thomson \& Wright 1990; Thomson 1991). A few models invoke internal mechanisms for shell formation such us gas ejection powered by either the central AGN or supernova explosions (Fabian et al. 1980; Williams \& Christiansen 1985). In the accretion model, shells are density waves formed by the infall of stars from a companion. The accretion/merger mechanisms qualitatively reproduce the basic characteristics, such as spatial distribution, frequency, and shape of observed shell systems (see e.g., Wilkinson et al. 2000; Pierfederici \& Rampazzo 2004; Sikkema et al. 2007, and references therein). The inner mechanisms cannot correlate the shell formation with the environment, whereas the accretion/merger models can explain why shells are predominantly found in low density environments (see Malin \& Carter 1983). Low-density environments can indeed favour the occurence of merger/accretion events, because the group velocity dispersion is of the order of the internal velocity of the member galaxies (Aarseth \& Fall 1980; Barnes 1985; Merritt 1985).

Observations in the past two decades have proven that a significant fraction of early-type galaxies contain gas in the ionized, atomic, and molecular phases. The study of the cold and warm gas phases provides new insight into the evolution of shell galaxies. Rampazzo et al. (2003) found that the warm ionized ( $\mathrm{H} \alpha$ ) gas and stars often appear dynamically decoupled indicative of external acquisition of the gas, as predicted by merger mechanisms (Weil \& Hernquist 1993). In the shell galaxy IC 4200, Serra et al. (2007) showed that the ionized gas is decoupled from stars and its rotation might be a continuation of the HI velocity field. In contrast, in other shell galaxies, e.g., NGC 2865, a clear association between the cold (HI/CO) gas and the kinematics of stars is evident, which appears to be inconsistent with the merger picture (Schiminovich et al. 1994, 1995; Charmandaris et al. 2000; Balcells et al. 2001).

The pioneering study of Schweizer \& Seitzer (1992) showed that, on average, field early-type galaxies with fine structures are significantly younger ( $\sim .6 \mathrm{Gyr})$ than those without ( $\sim 8 \mathrm{Gyr})$. The presence of fresh gas could lead to "rejuvenations" of the stellar populations, which may be used to estimate the time and duration of the polar ring and shell phenomena. The star formation history of shell early-type galaxies was analysed by Longhetti et al. (2000) using line-strength indices. They showed that shell-galaxies have a wide range of ages inferred from the
$\mathrm{H} \beta$ versus MgFe plane, which implies that recent and old interaction/acquisition events are equally probable. If shells formed at the same time as the "rejuvenating" event took place, shells ought to be long-lasting phenomena. Combining GALEX far UV data and line-strength indices, Rampazzo et al. (2007) showed that the peculiar position of some shell galaxies in the $(F U V-N U V)$ versus $\mathrm{H} \beta$ plane could be explained in terms of a recent (1-2 Gyr old) rejuvenation episode. Based on the linestrength indices, Serra et al. (2007) suggest that the origin of IC 4200, a HI-rich shell galaxy, was a major merger event that occurred 1-3 Gyr ago.

In this framework, we investigate the ability of GALEX $(F U V-N U V)$ colours to expand our view of stellar populations in galaxies that are sites of accretion/merger events such as the polar ring and shell galaxies. More specifically, for ring galaxies we estimate the age of the ring from the mix of stellar populations therein, while for shell galaxies we attempt to gain information about the "rejuvenation" process.

We present a study of shell galaxies that completes our Cycle 1 set of GALEX observations (ID = GI1-059). The UV observations include the polar ring galaxy MCG-05-07-001 and NGC 1210. We also include NGC 5329, which is in the list of galaxies with shells in the survey of Malin \& Carter (1983). These authors do not provide a description of the shell system, in contrast to most other cases in the catalogue, but note that NGC 5329 is located in a group $(\approx 10$ galaxies $)$ environment similar to that of both NGC 2865 and NGC 5018 studied by Rampazzo et al. (2007). This paper extends the UV study by Rampazzo et al. (2007). For this purpose, (1) we double the sample size of shell galaxies; (2) we consider shell galaxies with cold gas at their centre, enlarging the range of physical properties investigated; (3) we use detailed chemo-spectro-photometric models to study the colours of MCG-05-07-001 derived from the observations that extend from the far UV to near infrared.

The plan of the paper is as follows. Section 2 presents the sample and summarizes the current literature on the subject. Section 3 presents the observations. The main results of the observations are presented in Sect. 4. An attempt to interpret the results with particular emphasis on the $U V$ colours and their significance is given in Sect. 5. Finally, our conclusions are summarized in Sect. 6. We assume that $H_{0}=75 \mathrm{~km} \mathrm{~s}^{-1} \mathrm{Mpc}^{-1}$ throughout the paper.

\section{The sample}

Table 1 presents the main physical properties of our sample.

MCG-05-07-001, also known as ESO 415-G26, is a peculiar S0 galaxy (Whitmore et al. 1987; Iodice et al. 2002b). It is characterized by a polar ring less extended than the central host galaxy in the optical band. Debris is present in the galaxy outskirts at a position angle that is intermediate between the major axis of the host galaxy and the polar ring. Whitmore et al. (1987) reported observations of a "nice set of ripples and an extended asymmetric envelope". In attempting to date the accretion event responsible for the formation of the ring, they estimated an age of 1-3 Gyr. The HI map of this object was obtained with the VLA by van Gorkom et al. (1987), and van Gorkom \& Schiminovich (1997) noticed that the neutral hydrogen lies along the major axis of the polar ring, with some correlation between HI and outer shells. Based on the regularity of the HI distribution, van Gorkom et al. (1987) tried to estimate the age of the event generating the ring. In brief, they argued that the timescale for evenly distributing $\mathrm{HI}$ in an orbital plane must be 
Table 1. Relevant photometric, structural, and kinematic properties of MCG-05-07-001, NGC 1210, and NGC 5329.

\begin{tabular}{|c|c|c|c|c|}
\hline & "MCG-05-07-001 & NGC 1210 & NGC5329 & Ref. \\
\hline \multirow[t]{2}{*}{ Other identifications } & ESO 415 G26 & ESO 480- G 031 & \multirow[t]{2}{*}{ WBL 472-001 } & \\
\hline & AM 0226-320 & AM 0304-255 & & \\
\hline Morphological type & S0-pec & $\left(\mathrm{R}^{\prime}\right) \mathrm{SB}(\mathrm{rs})-\mathrm{pec}$ & $\bar{E}$ & [1] \\
\hline Mean Hel. Sys. Vel. $\left[\mathrm{km} \mathrm{s}^{-1}\right]$ & $4604 \pm 14$ & $3878 \pm 7$ & $7109 \pm 31$ & [1] \\
\hline Adopted distance $[\mathrm{Mpc}]$ & 61.4 & 51.7 & 94.8 & \\
\hline$B_{T}$ & $14.70 \pm 0.13$ & $13.46 \pm 0.19$ & $13.37 \pm 0.15$ & [1] \\
\hline \multicolumn{5}{|l|}{ Galaxy structure: } \\
\hline Average ellipticity & 0.65 & 0.59 & \multirow{5}{*}{0.48} & [2] \\
\hline PA [deg] main body & 22 & 117.7 & & [2] \\
\hline PA (ring) [deg] & 94.2 & & & [3] \\
\hline HI total mass $\left[M_{\odot}\right]$ & $5.6 \times 10^{9}$ & & & [4] \\
\hline $\mathrm{H}_{2}$ total mass $\left[M_{\odot}\right]$ & $2.4 \times 10^{9}$ & & & [5] \\
\hline \multicolumn{5}{|l|}{ Kinematic parameters } \\
\hline Vel.disp. $\sigma_{0}$ stars $\left[\mathrm{km} \mathrm{s}^{-1}\right]$ & $127 \pm 3$ & $219 \pm 27[6]$ & \multirow[t]{2}{*}{$262 \pm 22$} & {$[2]$} \\
\hline Max. rotation $V_{\max } \operatorname{star}\left[\mathrm{km} \mathrm{s}^{-1}\right]$ & $145 \pm 12$ & $>129[6]$ & & [2] \\
\hline
\end{tabular}

References: [1] NED http://nedwww.ipac.caltech.edu/; [2] HYPERLEDA http://leda.univ-lyon1.fr/; [3] van Gorkom et al. (1987); [4] Schiminovich et al. (1997); [5] Galletta et al. (1997); [6] Longhetti et al. (1998b).

proportional to the orbital time multiplied by the ratio of the circular velocity to the typical random velocity. Since this last factor is typically of the order of 10 , whereas the ratio of the Hubble time to the orbital time is more typically 100 , they suggest that the polar ring in MCG-05-07-001 is the result of a relatively recent event. Optical data tend to support the times scales derived from the HI. In particular, the stellar debris in the vicinity of MCG-05-07-001, may be indicative of an age younger than a few orbital times.

NGC 1210 is classified as a peculiar and barred S0. Its near-infrared luminosity profile agrees with the Sersic law with $0.28 \pm 0.01<1 / n<0.24 \pm 0.03$, i.e., is consistent with the canonical $1 / n=0.25$ corresponding to the de Vaucouleurs law (Brown et al. 2003). The galaxy kinematics and the line-strength indices were measured by Longhetti et al. (1998a,b). The high velocity rotation (see Table 1) supports the idea that the galaxy is a true $\mathrm{S} 0$. The $\mathrm{H} \beta$ value provided by Longhetti et al. (1998b) is unfortunately not corrected for emission infilling, although there is ionized gas ([OII]) in the galaxy center, as shown in their Table 1 . The analysis by Longhetti et al. (1999) of the so-called "blue line-strength" indices (e.g., CaII $[\mathrm{H}+\mathrm{K}]$ lines and $\Delta 4000$ ) suggests that the galaxy has a very young nucleus. NGC 1210 was observed in HI by Schiminovich et al. (2001). The HI emission forms a ring that includes the galaxy center.

NGC 5329 is a bright elliptical galaxy and the dominant member of a poor cluster of galaxies, WBL 472, consisting of six companion galaxies according to White et al. (1999). In contrast, both NGC 1210 (see e.g., Malin \& Carter 1983) and MCG05-07-001 are isolated galaxies. Brocca et al. (1997) found that the nearest companion of MCG-05-07-001, is PCG 9331, which is located at $\approx 17^{\prime}$ (302 kpc) from the galaxy with a velocity difference of $\approx 51 \mathrm{~km} \mathrm{~s}^{-1}$, well beyond five times the diameter of the galaxy $\left(6.1^{\prime}\right)$, a distance they consider as the limit for isolation.

To summarize, the galaxies in the present sample might plausibly represent different aspects of a common phenomenon, i.e., an accretion event. In the following paragraphs, we emphasize the similarities and differences between these objects and those observed with GALEX by Rampazzo et al. (2007).

MCG-05-07-001 and NGC 1210 have a rich reservoir of HI in their main body (see Table 1), in contrast to that found for NGC 2865, NGC 5018, and NGC 7135, the shell galaxies investigated by Rampazzo et al. (2007), where HI is mostly detected outside the main body of the galaxies. HI is also found in other late-type members of the group, and in the tidal structures. Theureau et al. (2007) did not detect HI in the direction of the NGC 5329 group.

In the sample of Rampazzo et al. (2007), no galaxy displays type 1 shells, i.e., systems of aligned shells (see e.g., Prieur 1990, for definitions and statistics). NGC 2865 and NGC 5018 have "all-round" shell systems that are known as type 2; 7 shells are detected in NGC 2865 and 10 in NGC 5018. While the type of shell system of NGC 5329 is unknown, Prieur (1990) classifies the shell system of NGC 1210 as type 2, and finds 11 shells, containing about $1.5-2 \%$ of the total luminosity of the galaxy. Type 2 shells are rather common as about $30 \%$ of shell galaxies show this structure (Prieur 1990). NGC 7135 (Rampazzo et al. 2007) has a type 3 system of shells since they are neither aligned (type 1) nor "all-round" systems (type 2). Following the description and images in Whitmore et al. (1987), (see their Fig. 7 panels $c$ and $d$ ), MCG-05-07-001 should also be considered as having type 3 shells. Indeed, shells are seen in the northeast at $63^{\prime \prime}$ and $88^{\prime \prime}$, and possibly in the West at $52^{\prime \prime}$. The major axis of the faintest material is misaligned at about 36 degrees with respect to the major axis of the SO component.

In addition to the shells, MCG-05-07-001 presents a polar ring. In their Fig. 2 (mid panel), Rampazzo et al. (2007) showed that the fine structure of NGC 2865 does not solely consist of shells but also a tidal structure (reminiscent of a ring) that crosses the entire galaxy in the east-west direction, nearly perpendicularly to the major axis of the galaxy and to the shell system. It is unclear whether this feature is connected to the formation of the shells. In the case of the polar ring in MCG-05-07-001, Whitmore et al. (1987) suggested that during the merger, the stellar component of the accreted companion is dispersed into the halo, whereas the gas clouds experience collisions and quickly settle onto a new disk or ring.

In this context, the analysis of the UV colours and the linestrength indices of NGC 2865 and NGC 7135 suggest that they both had recent bursts of stellar activity in their centers, possibly as a consequence of the interaction/accretion episode that triggered the shell formation. MCG-05-07-001 and NGC 1210 probably experienced a major accretion event. To cast light on these issues and in particular on the age of the merger event, with the aid of the GALEX FUV and NUV data, the optical line-strength indices, the near-infrared observations, and theoretical simulations of spectral energy distributions, magnitudes, and colours, 

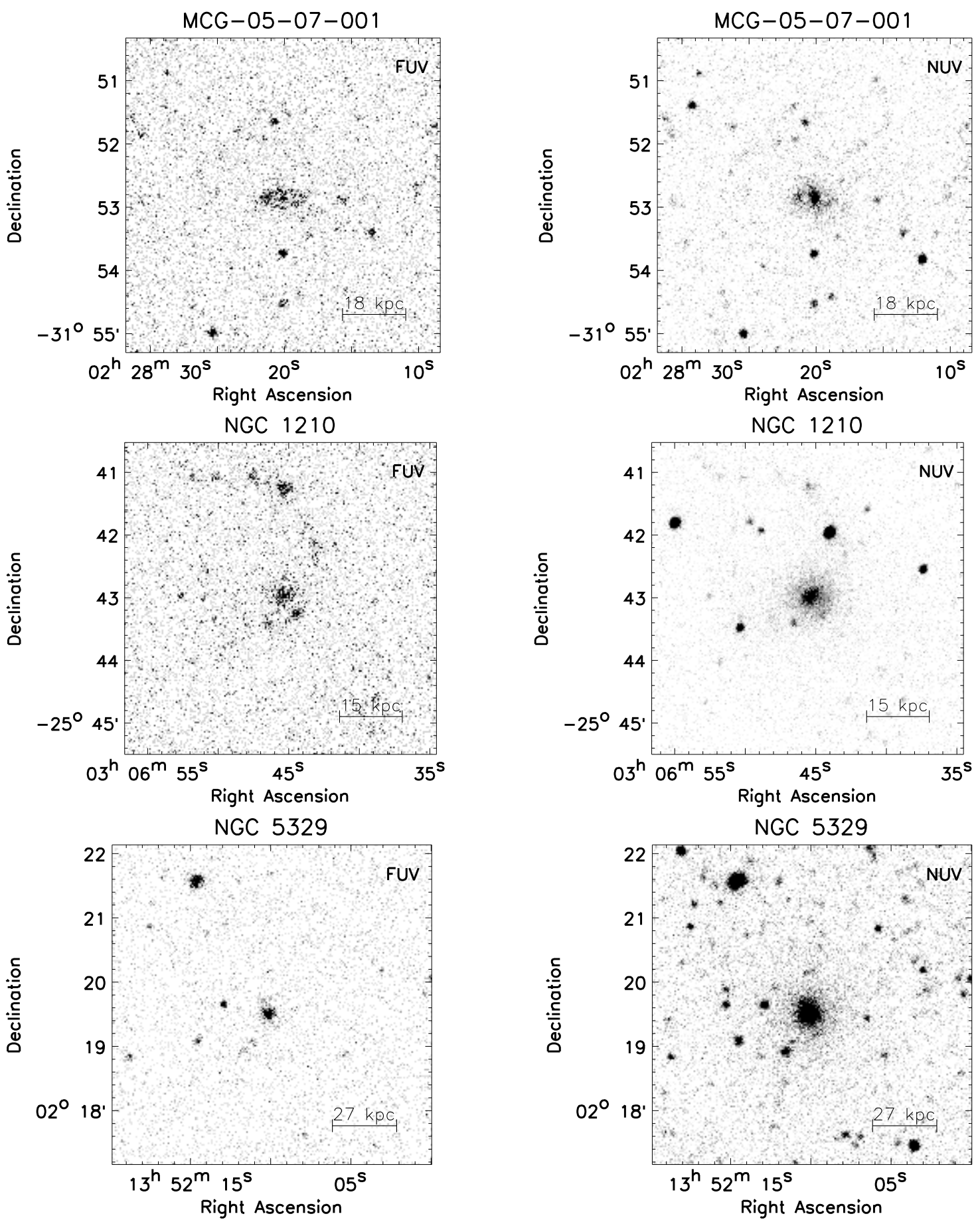

Fig. 1. Full resolution, $5^{\prime} \times 5^{\prime}$ wide, FUV (left panels) and GALEX NUV (right panels) background-subtracted images (counts $\left.\mathrm{px}^{-1} \mathrm{~s}^{-1}\right)$ of the three galaxies. The scale bar is derived from adopted distances provided in Table 1 .

we try to evaluate when in the galaxy history the wet accretion (i.e., gas rich) events took place. These estimates will be compared with timescales provided by morphological and kinematical considerations given in the literature to investigate the possible connection between rejuvenation episodes and the formation of the shell/rings.

\section{Observations}

The UV data were obtained with GALEX (see Martin et al. 2005; Morrissey et al. 2005, 2007), a $50 \mathrm{~cm}$ diameter modified Richey-Chrètien telescope with a very wide field of view
(1.2 degrees) and a high image spatial resolution $\approx 4$.' 5 and 6.' 0 $F W H M$ in FUV (1350-1750 ̊) and NUV (1750-2750 ̊) respectively, sampled with $1^{\prime \prime} .5 \times 1$ '. 5 pixels.

NGC 1210 and MCG-05-07-001 were observed during dedicated runs assigned to our team, whereas the data of NGC 5329 were taken from the GALEX archive (Medium Imaging Survey: MIS). The observing logs for each galaxy, including exposure times, are provided in Table 2 . The full resolution images in the $N U V$ and $F U V$ bands are shown in Fig. 1.

To the $G A L E X$ data, we add the $B$ - and $K$-band photometric data from Iodice et al. (2002b) for MCG-05-07-001 and the 

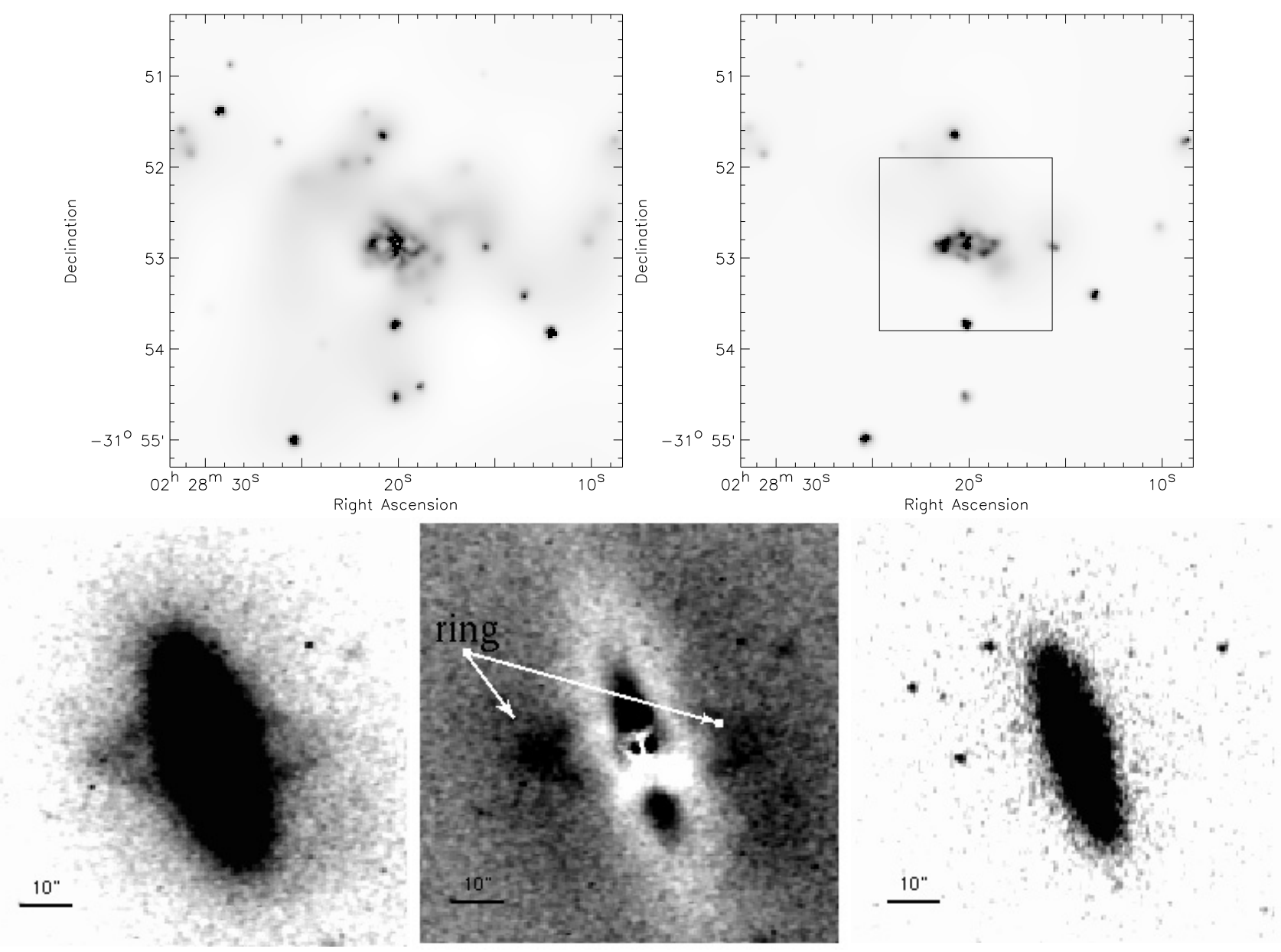

Fig. 2. GALEX UV images in a $5^{\prime} \times 5^{\prime}$ field around MCG-05-07-001. Top panels: NUV and FUV images obtained with ASMOOTH for $\tau_{\text {min }}=5$ and $\tau_{\min }=3$, respectively. The insert on the FUV image indicates the field of view of the B images. Bottom panels: B-band image (left) and $B$-band residuals with respect to the 2D model (middle), $K$-band image (right) (see also Iodice et al. 2002a). In the $K$-band image, the polar ring is not visible, whereas in the $B$-band and in the $F U V$ and $N U V$ bands the ring is the prominent feature. In the $B$-band residuals, darker colours correspond to regions where the galaxy is brighter than the model.

Table 2. Journal of the GALEX observations.

\begin{tabular}{|c|c|c|c|c|}
\hline Ident. & $\begin{array}{l}N U V \\
{[\mathrm{~s}]}\end{array}$ & $\begin{array}{l}F U V \\
{[\mathrm{~s}]}\end{array}$ & $\begin{array}{l}\text { Observing } \\
\text { date }\end{array}$ & P.I. \\
\hline MCG-05-07-001 & 1510 & 1531 & $2005-11-03$ & D. Bettoni \\
\hline NGC 1210 & 1558 & 1608 & 2005-11-04 & D. Bettoni \\
\hline NGC 5329 & 3869 & 2666 & 2004-06-09 & MIS \\
\hline
\end{tabular}

analysis of the Sloan Digital Sky Survey (SDSS) archival data in the $u$ [2980-4130 $\AA$ ], $g$ [3630-5830 ̊], $r$ [5380-7230 ̊], $i$ [6430-8630 ̊], and $z$ [7730-11230 ̊] bands for NGC 5329 (e.g., Stoughton et al. 2002).

CCD images of NGC 1210 in the Gunn $r$ band [6000-8500 A; central wavelength $6733 \AA$ A ] were taken from the European Southern Observatory, (ESO) Public Archive (prog: 68.B-0023). They were obtained with EMMI at ESO-NTT telescope with the red filter $R \# 773$. The detector used for the observations consisted of two MIT/LL CCDs. The average seeing had a $F W H M \approx 0 . ' 84 \pm 0 . ' 03$ for the Gunn $r$. Three exposures with this filter were available with an integration time of $200 \mathrm{~s}$. The raw frames were preprocessed using the standard IRAF tasks for debiasing and flat-fielding. The flat-field was performed using a normalized sky flat. Each CCD was reduced independently: the images were dithered and shifted to obtain a complete image of the galaxy and its surroundings. After applying debiasing and flat-fielding, the frames of each CCD were combined to filter out spurious contamination by cosmic rays.

\section{Morphology and photometry}

\subsection{Galaxy morphology}

To enhance the $\mathrm{S} / \mathrm{N}$ in the galaxy outskirts and bring out any possible faint structures in the UV emission, we follow the procedure outlined by Ebeling et al. (2006). The only parameter required by ASMOOTH is the desired minimal $\mathrm{S} / \mathrm{N}, \tau_{\min }$. For each individual pixel, the algorithm increases the smoothing scale until the $\mathrm{S} / \mathrm{N}$ within the kernel reaches a specified value. Thus, while the noise is suppressed very efficiently, the signal (locally significant at the selected $\mathrm{S} / \mathrm{N}$ level and carrying information about the underlying physical cause) is preserved on all scales. In particular, this method allows us to detect weak, extended structural features falling in noise-dominated regions (see Figs. 2-5).

MCG-05-07-001. The morphology of this galaxy changes from UV to NIR: the bulk of the NUV and FUV emission is concentrated in the polar ring (see Fig. 2 top panels) and the nucleus of the galaxy. The main body of the galaxy is visible in B- and $\mathrm{K}$-images (Fig. 2 bottom panels) but is hardly detectable in the $N U V$ and $F U V$ bands. In the $B$-band, both the central spheroid 

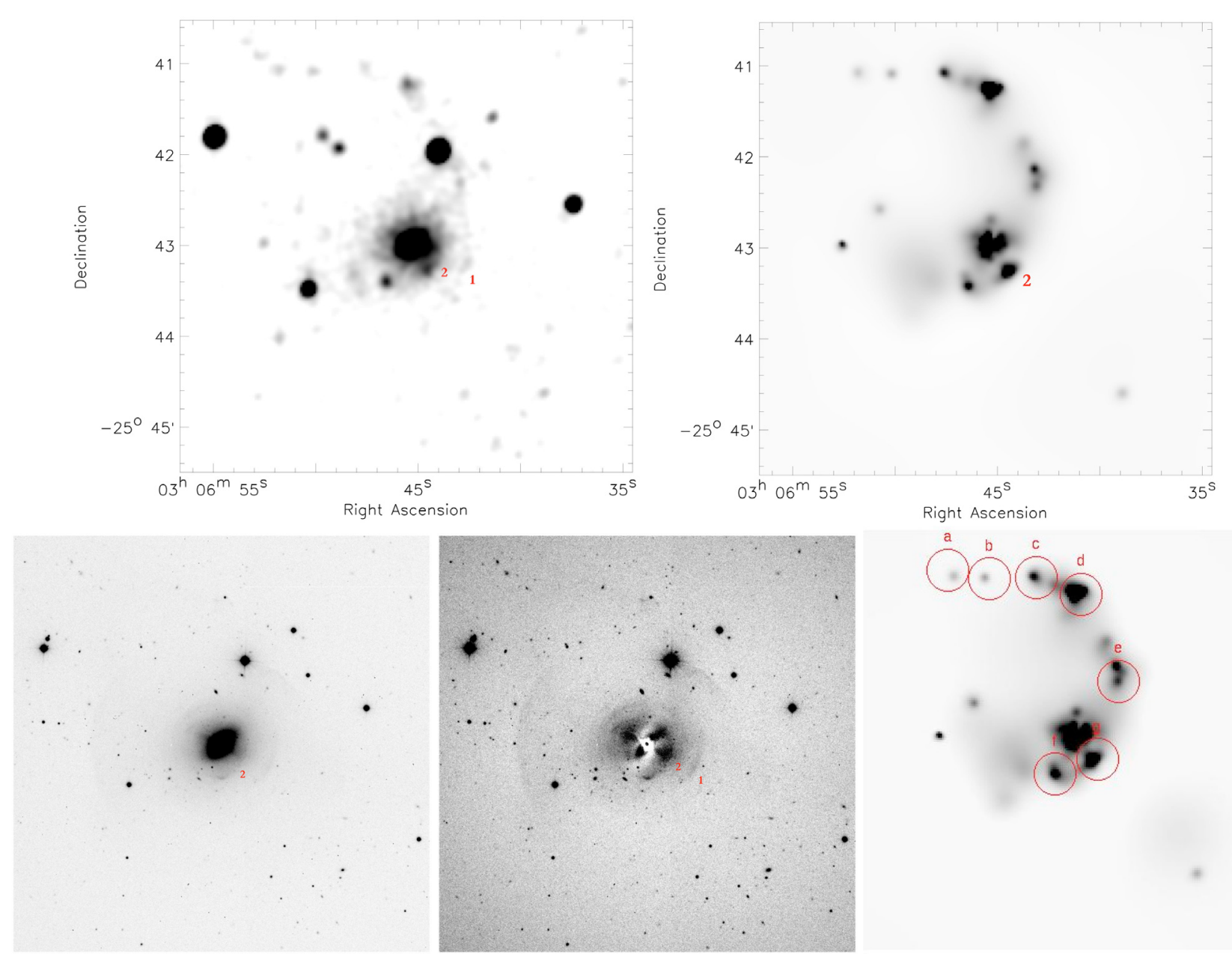

Right Ascension

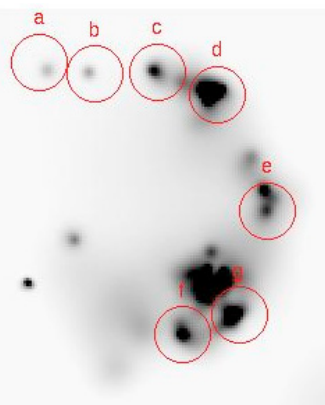

Fig. 3. Top panels: GALEX UV images in a $5^{\prime} \times 5^{\prime}$ field around NGC 1210 . The NUV image (on the left) was smoothed with an adaptive filter, whereas the FUV image (on the right) with ASMOOTH $\left(\tau_{\min }=3\right.$ ). Note how the system of debris overlays the HI emission in the bottom panel of Fig. 4. Bottom panels: NTT/EMMI uncalibrated $r$-band image in a $5^{\prime} \times 5^{\prime}$ (left) field and the same image where the main body of the galaxy has been subtracted revealing, together with shells, a complex central fine structure (mid panel). Labeled " 1 " and " 2 " are the two shell edges visible in the NUV and FUV images (see text). Superimposed on the smoothed FUV image, we indicate and label with letters the knots whose magnitudes are provided in Table 5 (right panel).

and ring contribute to the light and are clearly detectable; only the central spheroid contributes to the emission in the $K$-band; finally, the outer ring does not contribute significantly to the $K$ band thus becoming invisible (see also Iodice et al. 2002a). After applying ASMOOTH procedure to the NUV image, a large system of debris appears. We draw attention to the features northeast and northwest of the galaxy nucleus at a distance of about $1^{\prime}$ which lie at approximately the same distance and along the direction of the shell features detected by Whitmore et al. (1987) (see their Fig. 7, panels $c$ and $d$ ). An arc-like feature is also visible at $\approx 30^{\prime \prime}$ from the center towards the southwest direction in both the NUV and FUV images (in the latter, the feature is much weaker). The same can also be seen in panels $b$ of Fig. 7 in Whitmore et al. (1987). We performed a 2D model of the B-light distribution in the central spheroid using GALFIT and by masking the outer regions of the polar ring and foreground stars. The best-fit model implies that the central object is a S0 galaxy with an exponential bulge. This result is consistent with that derived by Iodice et al. (2002a) using NIR images (J, H, K). Subtracting the bestfit model from the B-image, the residuals are shown in Fig. 2 (middle panel): most of the polar ring structure (on the southeast and northwest sides) is very clearly distinctive. On the southwest side, the polar ring passes in front of the galaxy, thus the residuals show both the absorption by the dust (lighter regions) and the emission by the stars in the ring (darker regions). On the northeast side, where the ring passes behind the galaxy, only the excess of light from the stars in the ring is visible. Furthermore, the residual image detects an additional feature that encircles the entire galaxy and corresponds to regions where the model is brighter than the galaxy: this structure is also present in the NIR residuals (see Fig. 1 in Iodice et al. 2002b), where it has the same morphology but is much fainter, and in the NIR colour maps, where it appears bluer with respect to the inner regions of the galaxy (see Fig. 4 in Iodice et al. 2002b). This "cross-check" with the NIR images suggests that this feature could be real and not the result of an artifact of the fitting: it may indeed be an additional lens-like component.

NGC 1210. As for the previous galaxy, the NUV and FUV images (Fig. 3 top panels) show a different structure compared to that inferred from the EMMI/NTT $R$-band image (Fig. 3 left bottom panel). In the $N U V$ and $F U V$ passbands, the galaxy appears to consist of a nucleus, from which a long tidal tail departs toward the north direction, and a diffuse cloud of debris towards the south. Along this tail, several knots with a luminosity similar to that of the nucleus are visible. In the $R$ band, the galaxy exhibits a system of shells, that are more extended than the galaxy 

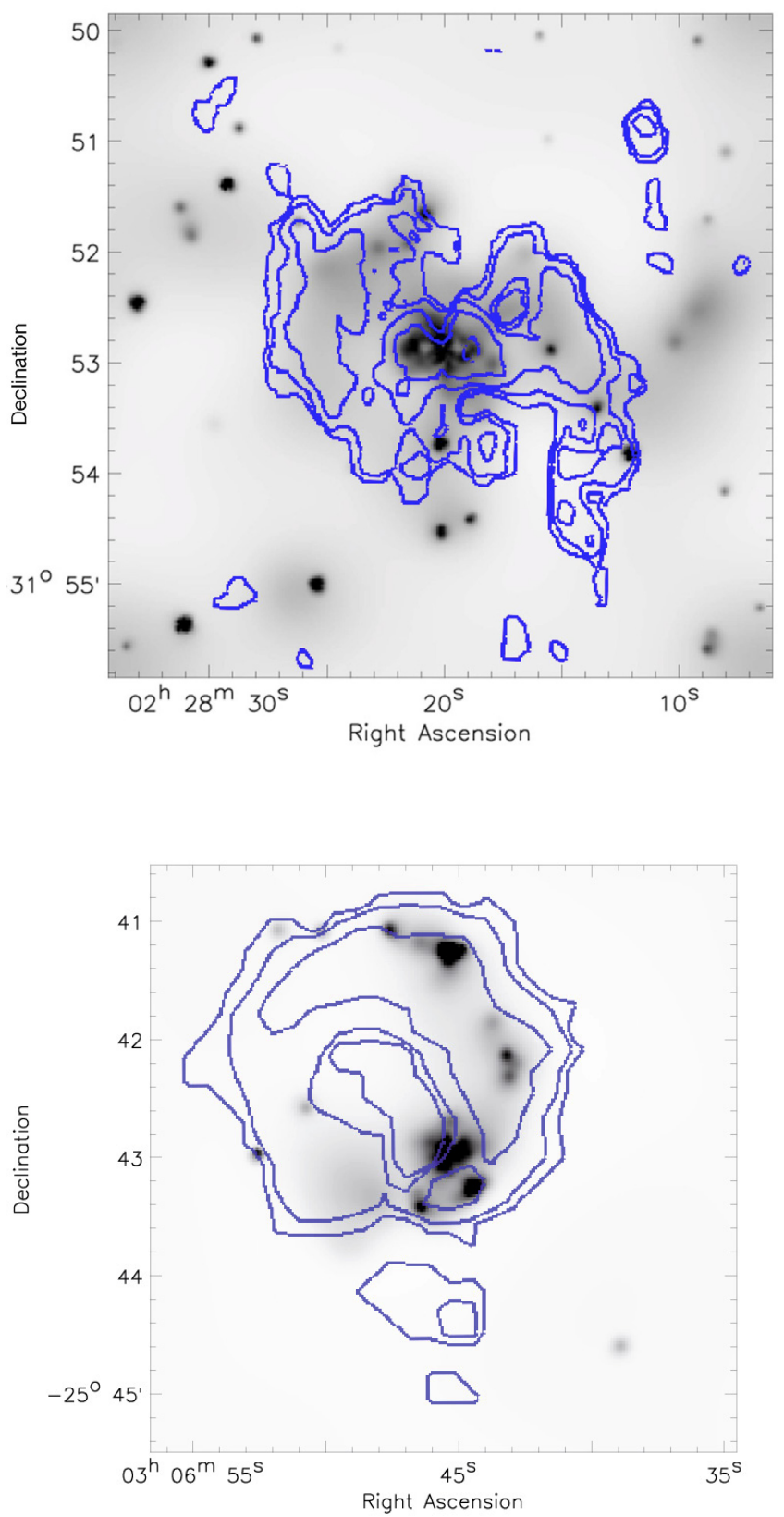

Fig. 4. Top panel: MCG-05-07-001. VLA B+C array 32". $1 \times 15^{\prime \prime} 2$ resolution HI observations performed by Schiminovich (2001) overlaid on the FUV image. The HI emission overlays the polar ring and the system of debris visible in the FUV image. Bottom panel: NGC 1210. VLA C + D array $52^{\prime \prime} \times 36^{\prime \prime}$ resolution observations (see Schiminovich et al. 2001). Part of the HI ring of NGC 1210 is delineated by strong FUV emission. The polar ring in MCG-05-07-001, and the northwest part of the HI ring in NGC 1210 have blue $(F U V-N U V)$ colours suggesting very recent star formation.

body. A faint, edge-on structure, perpendicular to the major axis of the galaxy, is visible in the inner part of the galaxy (see the left bottom panel of Fig. 4). Using GALFIT, we performed a $2 \mathrm{D}$ model fit to the galaxy light distribution in the $R$ band. Figure 3 (middle bottom panel) shows the model-subtracted image, revealing a complex fine structure composed of shells, edge-on structures perpendicular to the main galaxy body, and
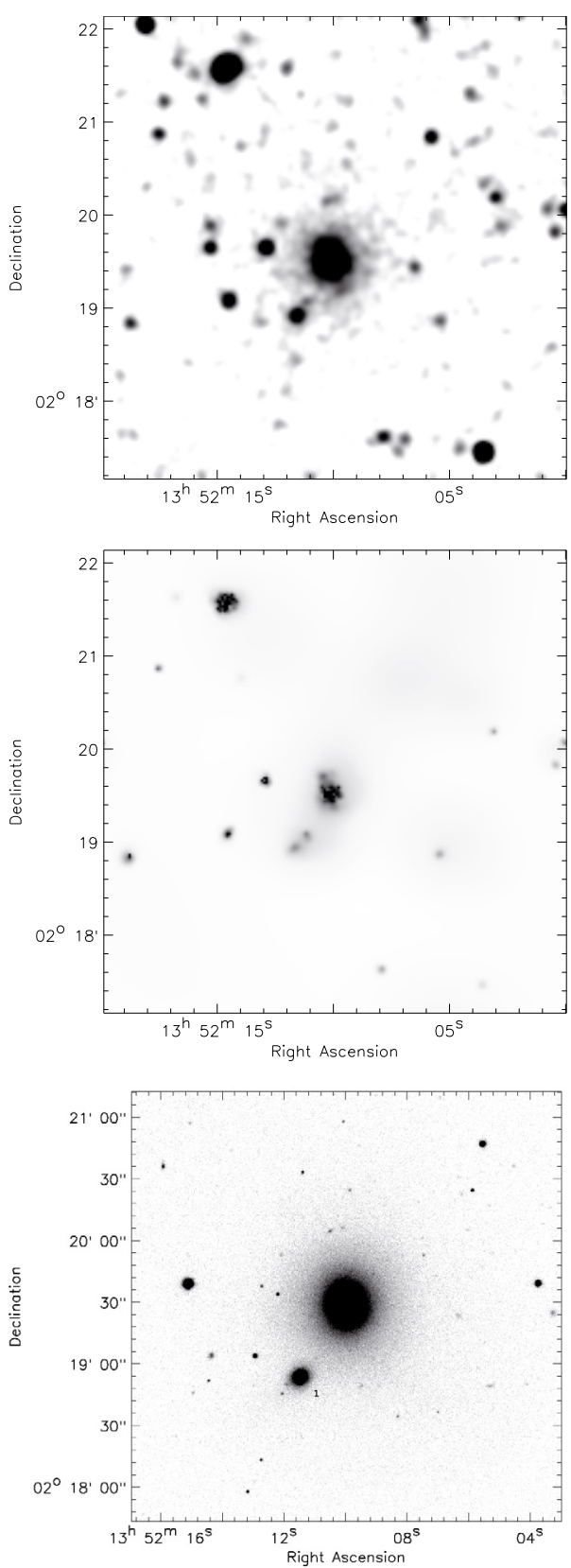

Fig. 5. GALEX UV images in a $5^{\prime} \times 5^{\prime}$ field around NGC 5329. Top and mid panels: NUV smoothed image and FUV image obtained with ASMOOTH for $\tau_{\min }=3$, respectively. Bottom panel: SDSS $\mathrm{r}$ image in $4^{\prime} \times 4^{\prime}$. The faint galaxy 2 MASX J13521155+0218545 is physically associated with NGC 5329.

dust (as often found in shell galaxies) (see e.g., Sikkema et al. 2007).

The west edges of the two inner shells are labeled 1 and 2 . Shell 1 is barely visible in the NUV image. The FUV image has two main features: (i) shell 2 is more luminous than shell 1; and (ii) there is a long tail of stars and gaseous material, which seems to be part of the same spiral-like structure extending towards the northeast. This suggests that shell 2 may have a different nature from shell 1 .

In Fig. 4, we show the HI distribution of MCG-05-07001 (top panel) and NGC 1210 (bottom panel) measured by Schiminovich (2001) and Schiminovich et al. (2001), respectively. These are overlaid onto the corresponding FUV images. For MCG-05-07-001, the HI distribution is elongated in the 
direction of the polar ring. Furthermore, for both MCG-05-07001 and NGC 1210 the HI distribution coincides with the system of debris visible in NUV and FUV emission. In particular, in NGC 1210 the HI emission forms a ring, which the tidal tail (detected by the GALEX FUV observations), belongs to.

NGC 5329. The NUV emission in NGC 5329 (Fig. 5, top panel) has an extension similar to that of the optical image (Fig. 5, bottom panel), whereas the FUV emission is concentrated in the central part of the galaxy (Fig. 5, middle panel). No peculiar structures, including shells, are detected in our optical image.

To summarize, NGC 5329 has the morphology of an elliptical galaxy in the $N U V$ bands. In the same bands, NGC 1210 shows a long tail, which is more clearly detected in the $F U V$ band. In MCG-05-07-001, the polar ring dominates both the NUV and FUV emission, whereas the nucleus can hardly be seen in these bands. A strong correlation exists between both the FUV and NUV emission and the HI distribution in MCG-05-07001 and NGC 1210.

\subsection{Light and colour distribution}

The surface photometry of these galaxies is based on the background-subtracted GALEX images and is calculated with the IRAF-STSDAS-ELLIPSE routine and the GALFIT package of Peng et al. (2002). The surface photometry profiles are derived from ELLIPSE, which considers the Fourier expansion of each successive isophote (Jedrzejewski 1987). Figure 6 shows the surface brightness and colour profiles of our galaxies. Furthermore, magnitudes, associated photometric errors, and colours, are derived from the original unsmoothed data in the GALEX $A B$ magnitude system. The zeropoints of $A B$ magnitudes are taken from Morrissey et al. (2007).

The disturbed morphologies of MCG-05-07-001 and NGC 1210 render the analysis of the surface brightness profiles more difficult than usual, especially in the UV. The NUV and FUV luminosity profiles map the nucleus and the ring of MCG05-07-001 (Fig. 6, top panel) and the bulge of NGC 1210 (Fig. 6, mid panel), respectively. In both cases, a strong variation characterizes the profile of the $(F U V-N U V)$ colour. The regular morphology of NGC 5329 has a smooth luminosity profile that follows the de Vaucouleurs $r^{1 / 4}$ law.

The $(F U V-N U V)$ colour maps, not shown in this paper, indicate that the nuclei of all the galaxies are red, with $(F U V-N U V) \simeq 1-1.5$ or even redder, probably because of dust absorption. In contrast, the knots in the polar ring of MCG05-07-001 (Fig. 2) and in the northern tail of NGC 1210 (Fig. 3) are blue, $(F U V-N U V \approx 0)$, which is indicative of a young component in the mix of the stellar populations. Table 3 summarizes the photometric data in the far UV and the structural properties of the three galaxies.

The B and K luminosity profiles of MCG-05-07-001 are much more extended than the UV light profiles, since this light is produced by the entire body of the galaxy. Figure 7 compares the $\mathrm{B}, \mathrm{K}$, and far-UV light profiles along the major axis of the main body of the galaxy, i.e., $\mathrm{PA}=22^{\circ}$. Colour gradients are visible not only in direction of the ring $\left(\mathrm{PA}=94^{\circ}\right)$ but in general from the centre to the periphery of the galaxy, since the nucleus is always redder than the outskirts.

The $U V$ and optical colour profiles of NGC 5329 (Fig. 6 bottom panel) are quite regular. Except for $F U V-N U V$, the nucleus is bluer in the other colour profiles than the galaxy outskirts. Given $(N U V-r)=5.6 \pm 0.02$ and $M_{r}=-22.05$, NGC 5329
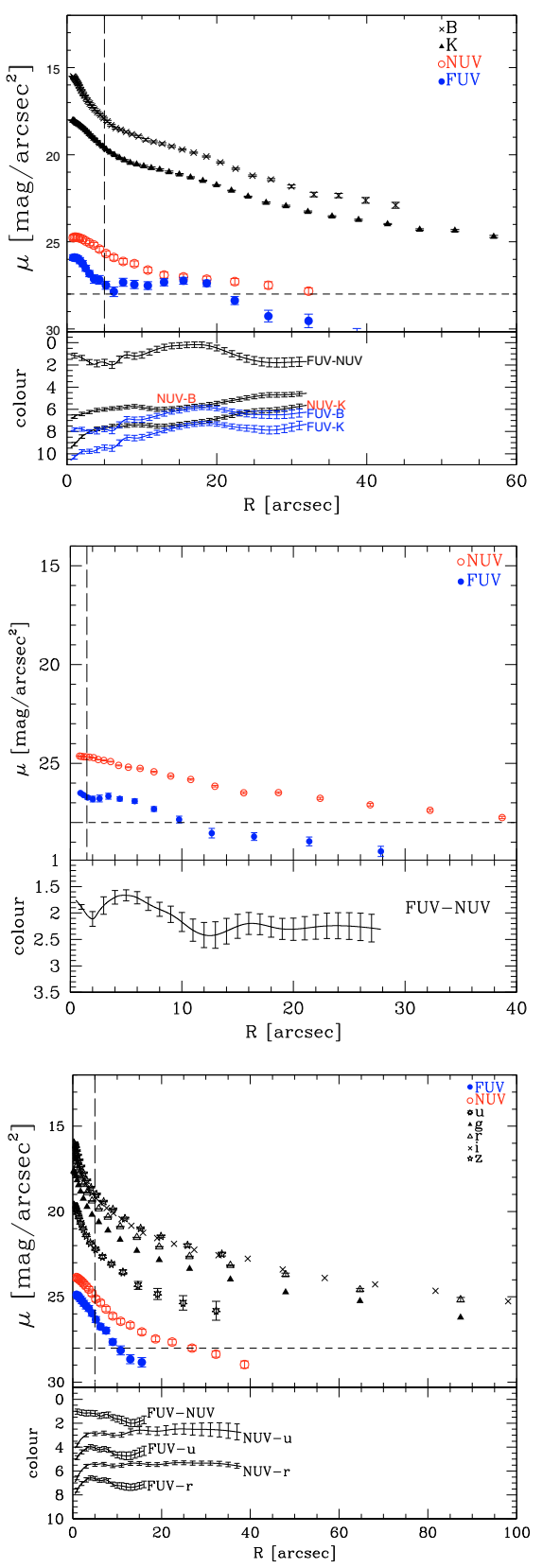

Fig. 6. Top panel: GALEX UV, B and K band (Iodice et al. 2002b) surface brightness of MCG-05-07-001. Mid panel: GALEX NUV and FUV surface brightness of NGC 1210. Bottom panel: GALEX UV and SDSS $u-g-r-i-z$ bands surface brightness profiles of NGC 5329. The vertical dashed line at $5^{\prime \prime}$ indicates the approximate FWHM of the GALEX point spread function. The horizontal line indicates the nominal UV surface brightness limit.

Table 3. Summary of the GALEX photometric data.

\begin{tabular}{lccc}
\hline \hline & MCG-05-07-001 & NGC 1210 & NGC 5329 \\
\hline$r_{\mathrm{e}}^{\mathrm{NUV}}[\operatorname{arcsec}]$ & 19 & 17 & 12 \\
$r_{\mathrm{e}}^{\mathrm{FUV}}[\operatorname{arcsec}]$ & 19 & 5 & 5 \\
$m_{\mathrm{FUV}}^{\text {tot }}(1530 \AA)$ & $19.78 \pm 0.07$ & $19.46 \pm 0.08$ & $20.20 \pm 0.04$ \\
$m_{\mathrm{NUV}}^{\text {tot }}(2310 \AA)$ & $18.67 \pm 0.07$ & $17.41 \pm 0.04$ & $18.20 \pm 0.02$ \\
$(F U V-N U V)^{\mathrm{tot}}$ & $1.11 \pm 0.10$ & $2.05 \pm 0.09$ & $2.02 \pm 0.05$ \\
\hline
\end{tabular}



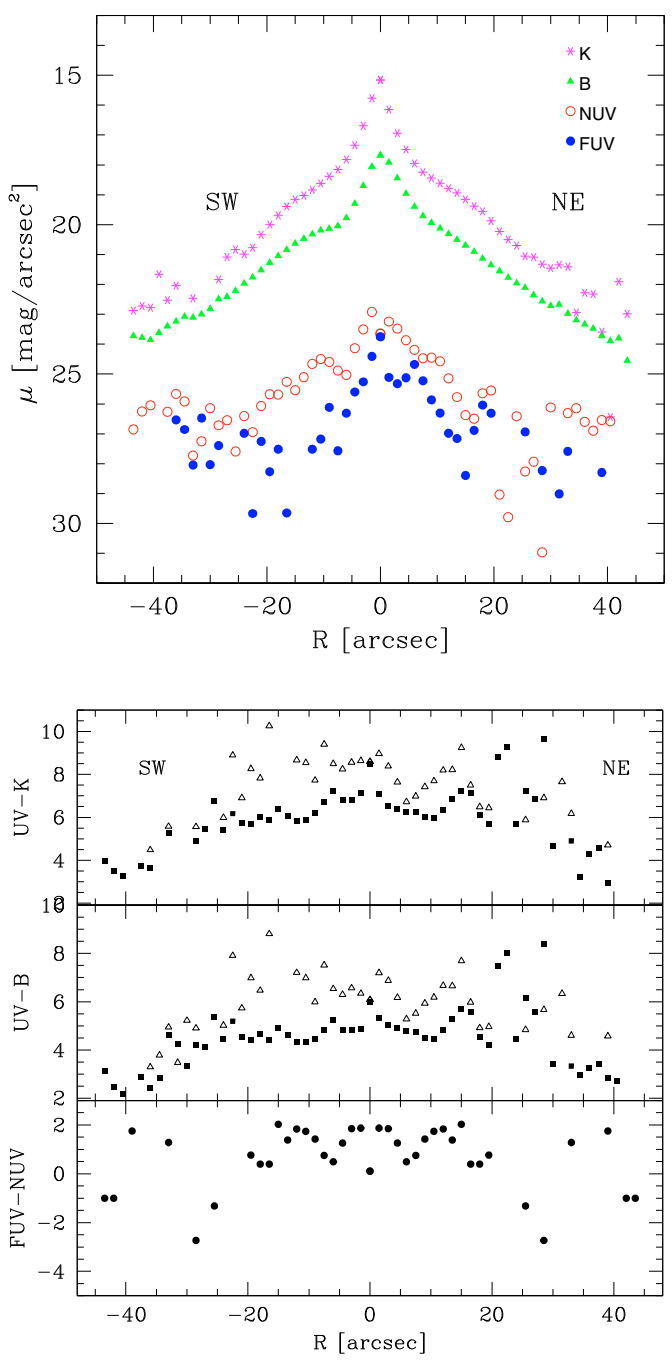

Fig. 7. Top panel: UV, B and $\mathrm{K}$ light profiles along the major axis of MCG-05-07-001 $\left(\mathrm{PA}=22^{\circ}\right)$. The average uncertainty is 0.05 and 0.04 for $B$ and $K$ bands respectively. Bottom panel: colours profiles along the MCG-05-07-001 major axis. The triangles indicate the $F U V-B$ and $F U V-K$ colours; the squares show the $N U V-B$ and $N U V-K$ colours.

is located on the red sequence of the (NUV-r) versus $M_{r}$ colourmagnitude diagram of Salim et al. (2007) (see their Fig. 1).

\section{Theory versus observations: a possible interpretation}

\subsection{How recent are the accretion events?}

The $U V$ colours are particularly suited to detecting very young stellar populations inside interacting galaxies (see e.g., Hibbard et al. 2005). However, some issues, need to be discussed when dealing with $U V$ colours.

Firstly, the possibility of high-metallicity, old stars (age $\geq 10 \mathrm{Gyr}$ and metallicity $Z \simeq 3 Z_{\odot}$ ) being present in early-type galaxies, even in small amounts, and could affect the $(F U V-N U V)$ colours. Indeed, the evolved stars in metalrich populations (extremely hot-HB and AGB-manqué stars) are widely accepted sources of the UV excess in quiescent earlytype galaxies (Bressan et al. 1994). Therefore, all models designed to reproduce the $(F U V-N U V)$ colours should consider that these high-metallicity stars are present, in particular when folding together young and old stellar populations. If some recent star formation activity adds young stars to a dominant older stellar population making the $U V$ colour blue, one should, before drawing any conclusion about the young stellar component, correct the $U V$ colours for the contribution of very old stars, which will not be negligible.

Secondly, our galaxy sample could exhibit strong morphological peculiarities such as polar rings and shells, customarily attributed to recent accretion/merging episodes that could have induced some star formation (see e.g., Longhetti et al. 2000; Rampazzo et al. 2007). It is then natural to believe that their stellar populations should span a range of ages, i.e., young stars could be present and thus contribute, in different proportions, to both the NUV and FUV fluxes.

Additionally in star-forming regions, the UV-optical stellar fluxes are strongly obscured by dust. The radiation in the UVoptical range is absorbed, and the effect is stronger at shorter wavelengths (Draine 2003), and re-emitted at longer wavelengths in the MIR/FIR. Therefore, dust can strongly affect the observed UV/optical magnitudes and colours and alter the shape of the SED. Modelling galaxies with star-forming regions requires an accurate treatment of dust, because of the combined extinction/emission effects.

Finally, the comparison of data with theory is made assuming that the complex stellar mix of a real galaxy can be reduced to a SSP of suitable metallicity and age. However, this approximation has different implications for the two parameters. While the metallicity distribution can be "reasonably" approximated to the mean value, the same does not hold for the age, when this value is derived from integrated properties (see e.g., Serra \& Trager 2007). In the discussion below, one has to keep in mind that the age that we are measuring from colours (and/or indices) is always biased by the last episode of star formation. In other words, it is a mean luminosity-weighted age, in which the most recent star-forming episode dominates at least during the first 2-3 Gyr from its occurrence. This is simply because of the rather well established law of luminosity fading of stellar populations, which ultimately mirrors the lifetime and evolutionary rate of a star as a function of its mass. So the ages derived adopting theoretical models represent lower limits to the age of the star-forming event (by accretion or whatever).

With the above points in mind, we apply the population synthesis technique to interpret our observational data and infer the secular evolution of our galaxies.

In the present study, only NGC 1210 has measured values of $\mathrm{Mg} 2(0.248 \pm 0.016)$ and $\mathrm{H} \beta$ (1.44) line-strength indices (Longhetti et al. 2000). The broad-band colours estimated for NGC 1210 are $(F U V-N U V)=2.09 \pm 0.40,(F U V-V)=$ $7.38 \pm 0.28$, and $(N U V-V)=4.43 \pm 0.73$.

In the case of MCG-05-07-001 and NGC 5329, we must adopt a different strategy based on the modelling of UV GALEX, optical, and NIR colours measured in suitable apertures. For MCG-05-07-001, we computed magnitudes within three apertures, of $r_{\mathrm{e}} / 4$ radius, located on the galaxy nucleus and north and south of the galaxy nucleus along the major axis (PA $\left.22^{\circ}\right)$. We further compute the magnitude within two apertures (of $r_{\mathrm{e}} / 2 \mathrm{ra}-$ dius) on both sides, east and west, of the ring (PA 94 ${ }^{\circ}$ ). The magnitudes calculated with different apertures for MCG-05-07-001 are given in Table 4.

Since NGC 5329 does not show peculiar features in its FUV images and its emission mainly originates in its central region, we calculated magnitudes centered on the nucleus within an aperture of $14^{\prime \prime}$ diameter, to match the 2MASS measurements and therefore extend the data-set from FUV to NIR. 
Furthermore, along the tidal tail of NGC 1210 there are knots as blue as $(F U V-N U V) \approx 0.2$, but no obvious counterparts are visible in the optical frame (see Fig. 3). Table 5 presents the magnitudes and colours of the knots, whereas Fig. 3 (bottom right panel) displays their position. We checked, using stellar catalogues, that no known stars contaminate the knots.

To interpret the properties of NGC 1210, we adopt the same photometric tool (stellar models and isochrones, library of stellar spectra, and the $A B$ mag photometric system) that has been used to calculate theoretical GALEX FUV and NUV magnitudes for SSPs in Rampazzo et al. (2007). We are aware that while the Lick line-strength indices (definited to be the ratio of a central bandpass and two pseudo-continuum bandpasses on either side of the central band, all bandpasses being a few tens of $\AA$ ) are basically insensitive to dust attenuation, which could affect the broad-band colours. The effect on ages estimated from ( $F U V-$ $N U V)$ and $(U V-V)$ colours, is discussed in the next paragraphs.

We first derive the age and metallicity dependence of the line-strength indices and ultraviolet colours and apply it to NGC 1210. Specifically, we study (i) the relationship between $\mathrm{Mg} 2$ and both $(F U V-V)$ and $(N U V-V)$ colours (see Fig. 8); and (ii) the diagnostic plane $\mathrm{H} \beta$ vs. $(F U V-N U V)$ colour, for the particular case of a $10 \mathrm{Gyr}$ old galaxy that experienced a recent burst of a star formation (see Fig. 9). Looking at the position of NGC 1210 in the two diagnostic planes of Fig. 8, the stellar content of this galaxy is compatible with a young age of about 2 $4 \mathrm{Gyr}$ and a mean metallicity in the range $0.04<Z<0.07$. The idea that the whole stellar content of NGC 1210 can be as young as 2-4 Gyr is perhaps too extreme. So it is worth exploring another possibility: the bulk of the stellar content of the galaxy is old but it suffered a recent burst of star activity and the age we infer is essentially that of the young component. This is the motivation for the experiment shown in the diagnostic plane $\mathrm{H} \beta$ versus (FUV-NUV) of Fig. 9 in which we combine an old population with a young one and follow the temporal evolution of the composite system. Examining Fig. 9 in some detail, the position of the 10 Gyr old model galaxy is shown by the empty star in the top left corner. The past evolutionary path of the old object is indicated by a solid line along which the arrow labelled "age" is drawn. If a burst of activity is assumed to occur, the path followed by the model galaxy is a sort of loop from the 10 Gyr position back to a new position very close to it after a certain time of the order of about $3 \mathrm{Gyr}$. The path is clockwise. The various curves correspond to different choices for the mean metallicity and relative amount of mass engaged in the novel stellar activity as indicated in the insert. Once more, the position of NGC 1210 is compatible with a young age that we now interpret as probably being caused by burst of star formation of a certain intensity, even if the possibility that the entire stellar content is really young cannot be excluded. NGC 1210 indeed lies close to the fading line of the prototype SSP at an age of about 4 Gyr.

To conclude, the positions of the nucleus of NGC 1210 in the planes Mg2-(FUV-V), Mg2-(NUV-V), and H $\beta$-(FUV-NUV) (Figs. 8 and 9) suggest that it hosts a young stellar population, which probably formed during the accretion episode. In Longhetti et al. (2000), the value of $\mathrm{H} \beta$ was not corrected for emission. The correction for the presence of an emission component within the absorption line would move the $\mathrm{H} \beta$ toward higher values, i.e., towards a position similar to that of NGC 2865 suggesting the contribution of a young ( $\leq 2$ Gyr old) stellar population.

The above estimate of age (and metallicity) of the young stellar component does not take the presence of dust into account;
Table 4. Magnitudes of the selected circular regions on MCG-05-07001 .

\begin{tabular}{lccccc}
\hline \hline Band & $H G_{\mathrm{c}}$ & $H G_{\mathrm{N}}$ & $H G_{\mathrm{S}}$ & $P R_{\mathrm{E}}$ & $P R_{\mathrm{W}}$ \\
\hline$F U V$ & $22.50 \pm 0.15$ & 25.32 & 24.41 & 21.55 & 22.40 \\
$N U V$ & $21.07 \pm 0.06$ & 23.17 & 22.30 & 21.24 & 21.61 \\
$B$ & $15.09 \pm 0.02$ & 17.64 & 17.57 & 17.56 & 17.75 \\
$J$ & $13.63 \pm 0.03$ & 16.49 & 16.34 & 16.28 & 16.36 \\
$H$ & $13.30 \pm 0.03$ & 16.32 & 16.32 & 16.76 & 17.10 \\
$K$ & $13.13 \pm 0.02$ & 16.18 & 16.25 & 16.77 & 17.06 \\
\hline
\end{tabular}

Note: the radius of the circular regions $P R_{\mathrm{E}}$ (East) and $P R_{\mathrm{W}}$ (West) is $r_{\mathrm{e}} / 2$ whereas the radius of $H G_{\mathrm{c}}$ (center), $H G_{\mathrm{N}}$ (North), $H G_{\mathrm{S}}$ (South) on the galaxy body is $r_{\mathrm{e}} / 4, r_{\mathrm{e}}$ being the galaxy effective radius. The typical errors are provided in Col. 1.

Table 5. Magnitudes of the knots along the tail of NGC 1210.

\begin{tabular}{cccc}
\hline \hline Region & $F U V$ & $N U V$ & $(F U V-N U V)$ \\
\hline a & $22.64 \pm 0.17$ & $21.36 \pm 0.07$ & $1.28 \pm 0.18$ \\
b & $22.75 \pm 0.17$ & $21.57 \pm 0.07$ & $1.18 \pm 0.19$ \\
c & $21.92 \pm 0.15$ & $21.58 \pm 0.07$ & $0.35 \pm 0.16$ \\
d & $20.92 \pm 0.12$ & $20.69 \pm 0.06$ & $0.22 \pm 0.13$ \\
e & $21.96 \pm 0.15$ & $20.75 \pm 0.06$ & $1.21 \pm 0.16$ \\
f & $21.86 \pm 0.14$ & $20.18 \pm 0.06$ & $1.68 \pm 0.15$ \\
g & $21.44 \pm 0.13$ & $19.64 \pm 0.05$ & $1.80 \pm 0.14$ \\
\hline
\end{tabular}

The position of the knots along the tail in NGC 1210 is shown in Fig. 3 (bottom right panel).

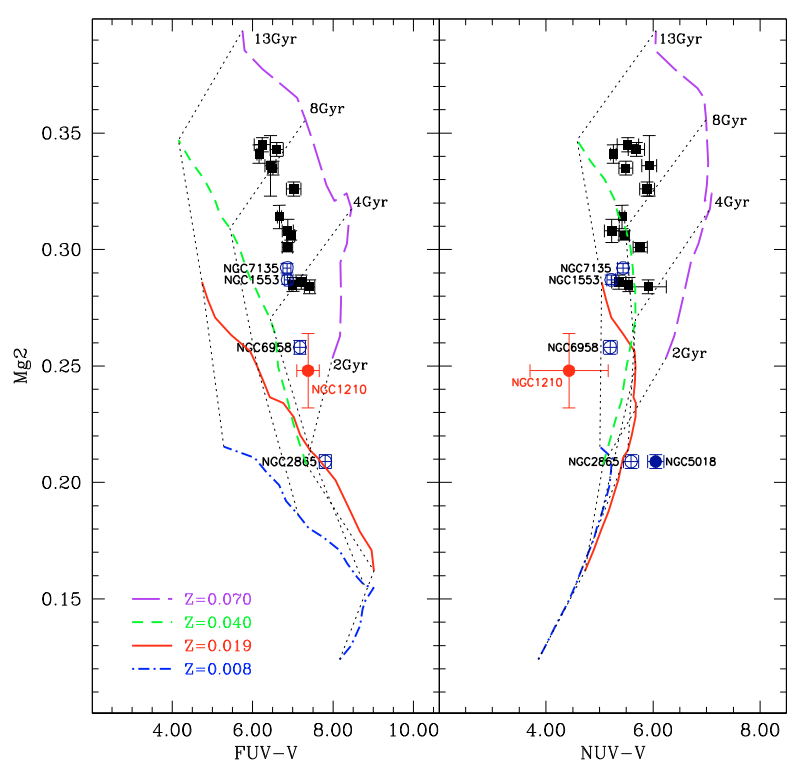

Fig. 8. Comparison of the theoretical relation $\mathrm{Mg} 2$ versus GALEX FUV (left) and NUV (right) - optical colours with observations. Dust-free SSPs of different age and metallicity are used. The loci of constant age and metallicity are shown as indicated. The data are from Rampazzo et al. (2007).

while the line-strength indices are not expected to change significantly in the presence of dust, the same does not apply to the broad band colours. Therefore, to properly analyse the effect of the dust attenuation on the age we need to use the library of SSPSEDs calculated by Piovan et al. (2006a) in which the effects of dust are taken into account. It is worth recalling here that there are at least three main circumstances in which dust influences the stellar light: (i) for a certain fraction of their life very young stars are embedded in the parental molecular clouds (MCs). Even if 


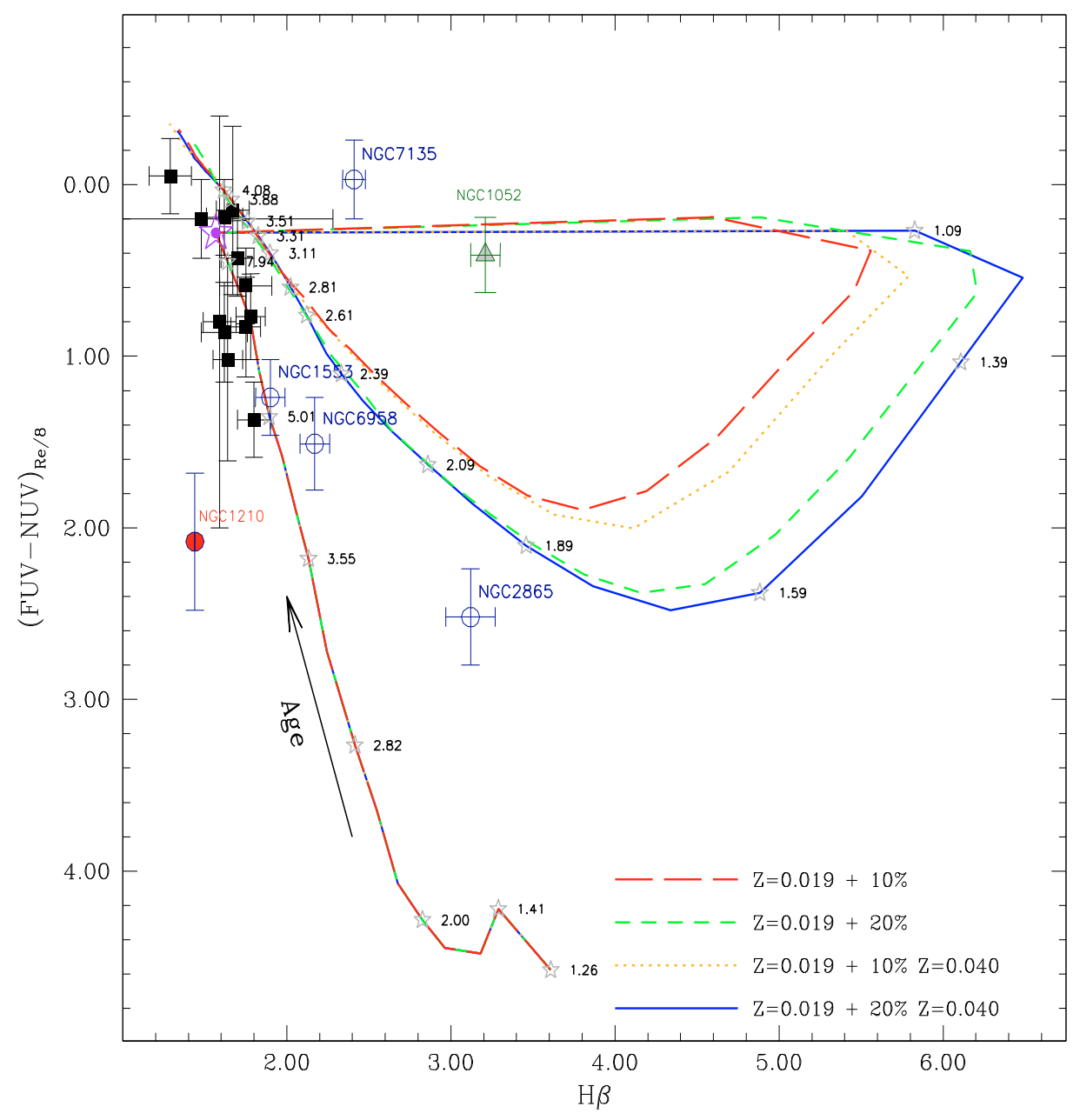

Fig. 9. GALEX $(F U V-N U V)$ colours, within an aperture of $r_{\mathrm{e}} / 8$ radius, versus $\mathrm{H} \beta$ of the sample of Rampazzo et al. (2007) and of NGC 1210 (red bullet). We also show the evolution of a SSP as a function of time (solid red line along which the arrow labelled age is drawn). The age increases as indicated by the arrow and a few values of the age in Gyr are marked. The position of a $10 \mathrm{Gyr}$ old galaxy of solar chemical composition is indicated by the large star located in the top left part of the plot. A burst of star formation, superimposed on such old stellar component, produces the "closed paths" traced in the plot by dotted, long-dashed, short-dashed, and solid lines according to the percentage of the mass involved in the burst and of the different metallicity in the newly born stars. The path starts from the empty stars at the top left, performs an extended loop, and goes back to a new position very close to the initial one. Several values of the age since the beginning of the burst are indicated. The true age of the composite SSP is $10+T_{\text {burst }}$ Gyr. Four combinations of the metallicity $Z$ and the percentage of star mass in the burst are considered as shown by the insert. Indeed a burst of activity can deeply change the $U V$ colour and the $\mathrm{H} \beta$ index of an underlying old population. Notice that the $\mathrm{H} \beta$ value was not corrected for emission-line infilling: the correction for the presence of an emission component within the absorption line would move the $\mathrm{H} \beta$ line-strength indices toward higher values (right side of the plot) toward the location of NGC 2865 . The Mg2 and $\mathrm{H} \beta$ line-strength indices values of NGC 1210 are taken from Longhetti et al. (2000).

the duration of this obscured period is short, because SNe explosions and stellar-wind energy injection sweep away the gas soon after the onset of star formation, its effect on the light emitted by these young stars cannot be neglected. Excluding old ellipticals, in which star formation stopped at relatively early epochs, for all morphological types the impact of young dusty populations on the galaxy SED has to be considered (see Takagi et al. 2003; Piovan et al. 2006a; Siebenmorgen \& Krügel 2007, for more details about young SEDs); (ii) low- and intermediate-mass stars in the asymptotic giant branch (AGB) phase may form an outer dust-rich shell of material obscuring and reprocessing the radiation emitted by the star underneath (see Bressan et al. 1998; Piovan et al. 2003; Marigo et al. 2008, for the relation between dusty shells around AGB stars and SSPs); (iii) finally, because of the contribution of metal-rich material by supernovae and stellar winds, the ISM acquires over the years a dust-rich diffuse component capable of absorbing/re-emitting radiation (see
Weingartner \& Draine 2001a; Draine \& Li 2001; Li \& Draine 2001, for models of extinction and emission of a dusty ISM).

The Piovan et al. (2006a) library of SSPs takes into account the effect of dust by parental MCs and AGB stars [items (i) and (ii) above]. It has been successfully employed to reproduce the SEDs of the active central regions of star-forming galaxies from the UV to the far-IR by varying some basic parameters. The parameter space of the library includes: metallicity $Z$ and age $T_{\mathrm{SSP}}$ of the SSP, and a number of dust-related parameters, namely the optical depth $\tau_{V}$ in the $V$-band, the abundance of carbon in the small carbonaceous grains $b_{\mathrm{C}}$ (Weingartner \& Draine 2001a), the scaling parameter $R$ (Takagi et al. 2003), and the ionization state of PAHs (Weingartner \& Draine 2001b). Since $R, b_{\mathrm{C}}$, and the ionization state affect the SED mostly at middle and far-IR wavelengths where we have no data available for our sample of galaxies, the only parameter of interest is $\tau_{V}$. This is chosen to correspond to the case of highly obscured, star-forming regions, 


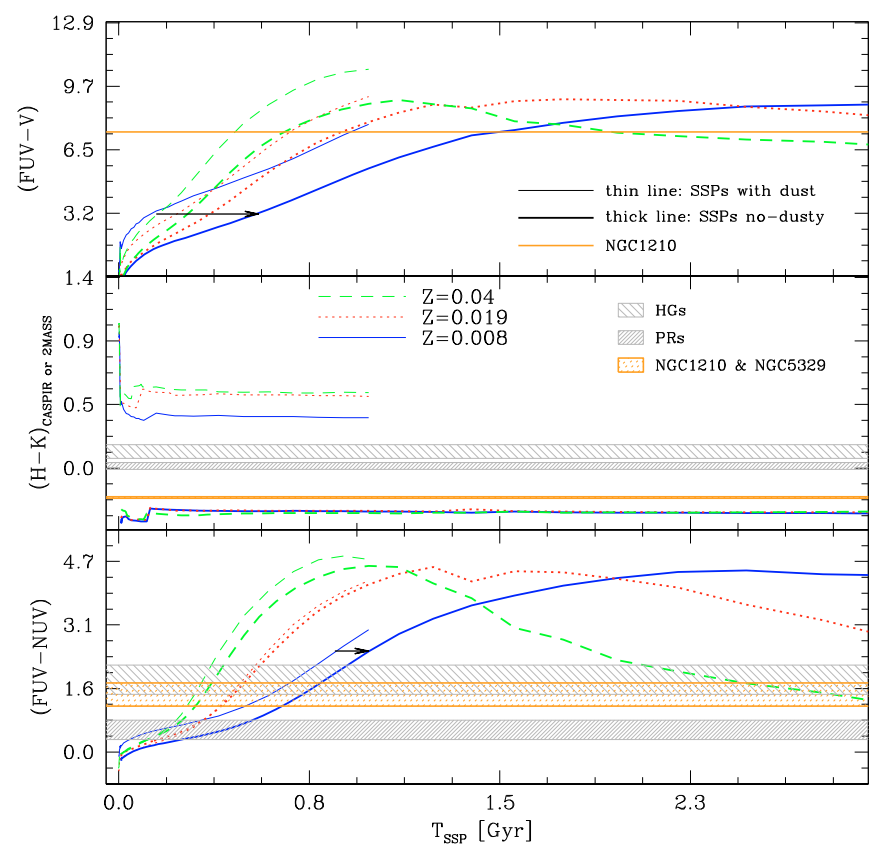

Fig. 10. The effect of dust on SSPs colours. The shaded bands represent the colours of NGC 1210, MCG-05-07-001 and NGC 5329. Top panel: evolution of the $(F U V-V),(H-K)$ and $(F U V-N U V)$ colours for "classical", dust-free SSPs (thick line) and dusty SSPs (thin line with $\tau_{V}=35$ (Piovan et al. 2006a)) both shown for three different metallicities: $Z=0.008,0.019$ (solar), and 0.04 (solid, dotted, and dashed lines, respectively). Bottom panel: the same but for the GALEX (FUV-NUV) colour. The arrows, in the top and bottom panels, indicate the "uncertainty" in the age estimate passing dusty to dust-free SSPs at given colours.

i.e., $\tau_{V}=35$ (Piovan et al. 2006a). The effects of both dust from self-contamination, parental molecular clouds in which stars are born, and stellar winds extend from very young ages to ages of about 1 Gyr. Of course, these SSPs may still be embedded in a diffuse interstellar component, which has to be treated separately, but at least for the self obscuration 1 Gyr is a safe upper limit to its age (see Piovan et al. 2006a,b, for a detailed explanation). With this new library, we (a) study the effect of dust extinction on the colours, at least in the age range 10 Myr to 1 Gyr and (b) study the effect of a burst of star formation (by dusty using SSPs) superimposed on an old dust-free SSP.

In Fig. 10, we show the evolution of the colours ( FUV $V),(F U V-N U V)$, and $(H-K)$ for both classical and dustySSPs as a function of different metallicities. The shaded bands indicate the observed colours of MCG-05-07-001, NGC 1210 and NGC 5329.

The attenuation of the $U V$ colours produced by dust introduces an uncertainty in the age that depends on the metallicity. For $(F U V-N U V)$ and $(F U V-V)$, the error is as large as $\sim 0.15$ Gyr and $\sim 0.5$ Gyr for a typical metallicity at $Z=0.008$ (arrows in the bottom and top panels of Fig. 10), respectively. In the mid panel of Fig. 10, we show the colour $(H-K)$ for classical and dusty SSPs as a function of the age and metallicity as indicated. The shaded band in the same panel show the $(H-K)$ colour measured for NGC 1210, NGC 5329, and MCG-05-07-001. To reproduce the NIR colours of the three subregions centered on the northern, central, and southern regions of the host galaxy MCG-05-07-001 and of the two regions centered on the western and eastern regions of the ring. Figure 10 shows that we have to introduce a significant amount of dust

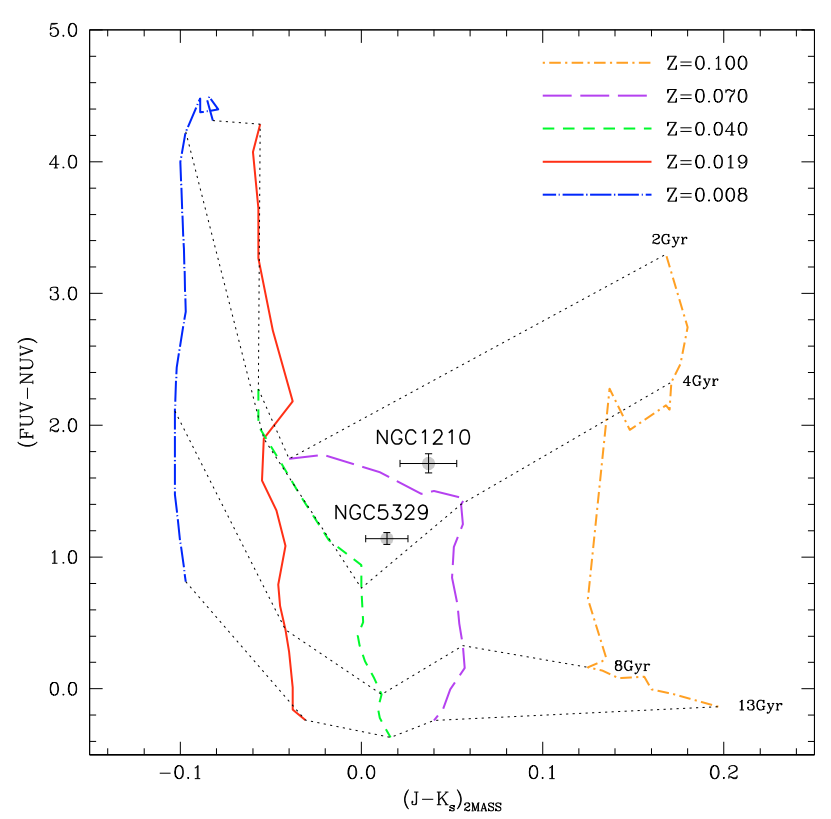

Fig. 11. Comparison between theoretical colour-colour relations and GALEX, optical, and NIR measurements. We plot the GALEX (FUV $N U V)$ vs. $\left(J-K_{\mathrm{s}}\right)$ theoretical relations for different metallicities. The loci of constant age are shown for a few selected age values. No recent bursts are considered. The data for the central regions of NGC 5329 and NGC 1210 suggest that both galaxies probably experienced a recent rejuvenation of their stellar populations.

in the SSPs. We conclude that the age estimation of galaxies in the $(F U V-N U V)-\mathrm{H} \beta$ (Fig. 9) as well as in the $\mathrm{Mg}_{2}-(\mathrm{FUV}-\mathrm{V})$ plane is certainly influenced by dust attenuation, the estimated age uncertainty is any a fraction of a Gyr (or smaller given that we consider high extinction).

In Fig. 11, we compare the GALEX $(F U V-N U V)$ and $\left(J-K_{\mathrm{s}}\right)$ colours of NGC 1210 and NGC 5329 with those predicted by dust-free SSPs. No recent burst of star formation is considered at this stage of the analysis. The theoretical loci of constant age and or metallicity are shown as indicated. According to mid panel in Fig. 10, the dust should introduce an age uncertainty (small) in the (FUV-NUV) colour and a large effect in the $(J-K)_{2}$ MASS colour. Since in Fig. 11, the age follows a trend nearly perpendicular to $(J-K)_{2 \text { MASS }}$ axis we suggest that this should translate into a large uncertainty in the galaxy metallicity rather than the age. Looking at the colours measured in the selected regions centered on the NGC 5329 and NGC 1210 nuclei, we may conclude that these galaxies probably experienced a recent ( $\sim 2-4 \mathrm{Gyr})$ rejuvenation of its stellar population. Although NGC 5329 seems to be a very relaxed elliptical in the far UV frames, the estimated luminosity weighted age of the nucleus, inferred from the data, suggests the presence of a young component in its stellar population.

For MCG-05-07-001, Iodice et al. (2002a), near-infrared analysis infer the presence of a very young ( $\leq 1 \mathrm{Gyr})$ stellar population (see their Figs. 2 and 3).

In Fig. 12, we analyse the effect of a secondary burst of star formation on the colour evolution. We use both dust-free and dusty SSPs to simulate old and recent stellar components. We consider two cases of star-formation bursts. The first case (left panels) involves $30 \%$ of the stellar population in the recent burst, and the other (right panel) involves $70 \%$ of the total in the burst. Since the bursting activity is accompanied by dust, the two cases represent two different situations as far as the total amount of 

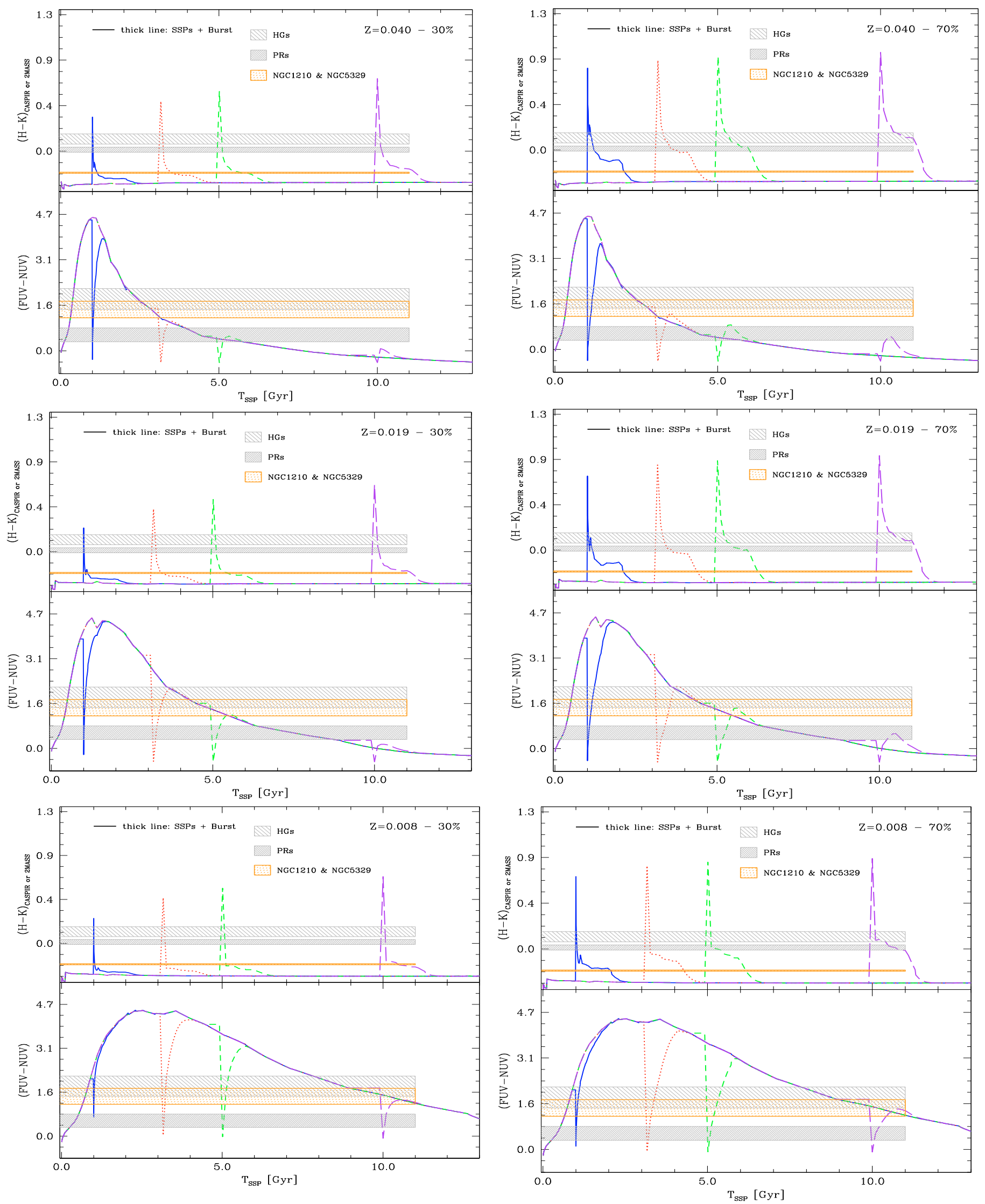

Fig. 12. Evolution of the $(H-K)$ and GALEX $(F U V-N U V)$ colours when a burst of star formation is superimposed on the passive evolution of a classical (dust-free) SSP. The observed colours of NGC 1210, NGC 5329, and MCG-05-07-001 in the host galaxy (HGs) and in the polar ring (PRs) are shown. The burst of star formation involves a certain percentage of the galaxy mass, namely 30\% (left panels) and of the 70\% (right panels). From, top to the bottom the burst is superimposed on a $10 \mathrm{Gyr}$ old SSPs with metallicities of $Z=0.04, Z=0.02$, and $Z=0.008$. 
dust is concerned. Furthermore, the second case can be considered as an upper limit in which most of the stellar activity occurs very recently. The shaded areas represent the observed colours of NGC 1210, NGC 5329, MCG-05-07-001 host galaxy (HGs), and polar ring (PRs). The following remarks can be made:

(1) The observed $(H-K)$ infrared colours can be reached by superimposing a burst of star formation. The NIR colours of both NGC 1210 and NGC 5329 probably can be produced by a weaker burst. The longer the period of activity or the stronger the burst, the longer will be the period during which the colours match the observed data. Since the colours of the underlying passive population are almost invariable, the effect of the burst does not depend on the burst onset time. Finally, there are no significant differences between the three metallicities.

(2) The GALEX (FUV - NUV) colour during the burst strongly depends on the metallicity. The higher the metallicity, the earlier the $(F U V-N U V)$ colour becomes close to the values given by the shaded regions of the passive evolution after a steep initial rise. Therefore, we can set an upper limit to the age at which the burst began, both for the HG and the PR regions. For the highest metallicity, the recent episode of star formation should have began some $<2-3$ Gyr ago (see the top panel of Fig. 12). For solar and sub-solar metallicities, the possible age of the secondary stellar activity is older (see the mid and bottom panels of Fig. 12). With these models, including the effects of dust, we interpret the UV, optical, and NIR colours of several selected regions of MCG-05-07001 (see Table 4).

To strengthen the above results, we consider the colour-colour relationships. In Fig. 13, we compare the theoretical two-colour plane GALEX $(F U V-N U V)$ and $\left(H-K_{\text {CASPIR }}\right)$ with the observational data for five selected regions of MCG-05-07-001. Two of them are centered on the eastern and western regions of the MCG-05-07-001 ring ( $\mathrm{PR}_{\mathrm{W}}$ and $\left.\mathrm{PR}_{\mathrm{E}}\right)$, whereas the remaining three are located on the northern, central, and southern regions of the host galaxy $\left(\mathrm{HG}_{\mathrm{N}}, \mathrm{HG}_{\mathrm{C}}, \mathrm{HG}_{\mathrm{S}}\right)$.

We plot the path of a recent burst of star formation $(0.5 \mathrm{Gyr}$ old) of different metallicity $Z=0.008,0.019$, and 0.04 and different intensity, namely corresponding to star formation within $30 \%$ (bottom panel) and $70 \%$ (top panel) of the galaxy mass. The SSP in use for the burst are those with dust by selfcontamination. The first choice for the burst intensity is based on the measured ratio of $\mathrm{H}_{2} /\left(\mathrm{H}_{2}+\mathrm{HI}\right)$ that could roughly indicate the amount of gas used up in star formation if stars are most likely to form in cold molecular clouds. With the second choice, we invert the percentages between dusty and dust-free SSPs and the composite population now involves a large fraction, 70\%, of young obscured stars. This latter choice represents a sort of upper limit where most of the stars are young and obscured.

As already found in Fig. 12, there are many possible ways of combining populations of different age, metallicity, and total mass. The particular cases shown in Fig. 13 refer to a metal-rich, relatively young stellar component to which a burst is added. The colour-colour diagram of Fig. 13 represents an extension in Fig. 12: different percentages of dusty SSPs are needed to reproduce the observed colours of PRs and HGs. In general, a burst makes colours redder in agreement with the observations. To conclude, we note that even the colour-colour diagnostic cannot unequivocally determine the age of the underlying stellar population: Figs. 12 and 13 clearly show that similar effects are produced by superposing a burst on an old metal-poor
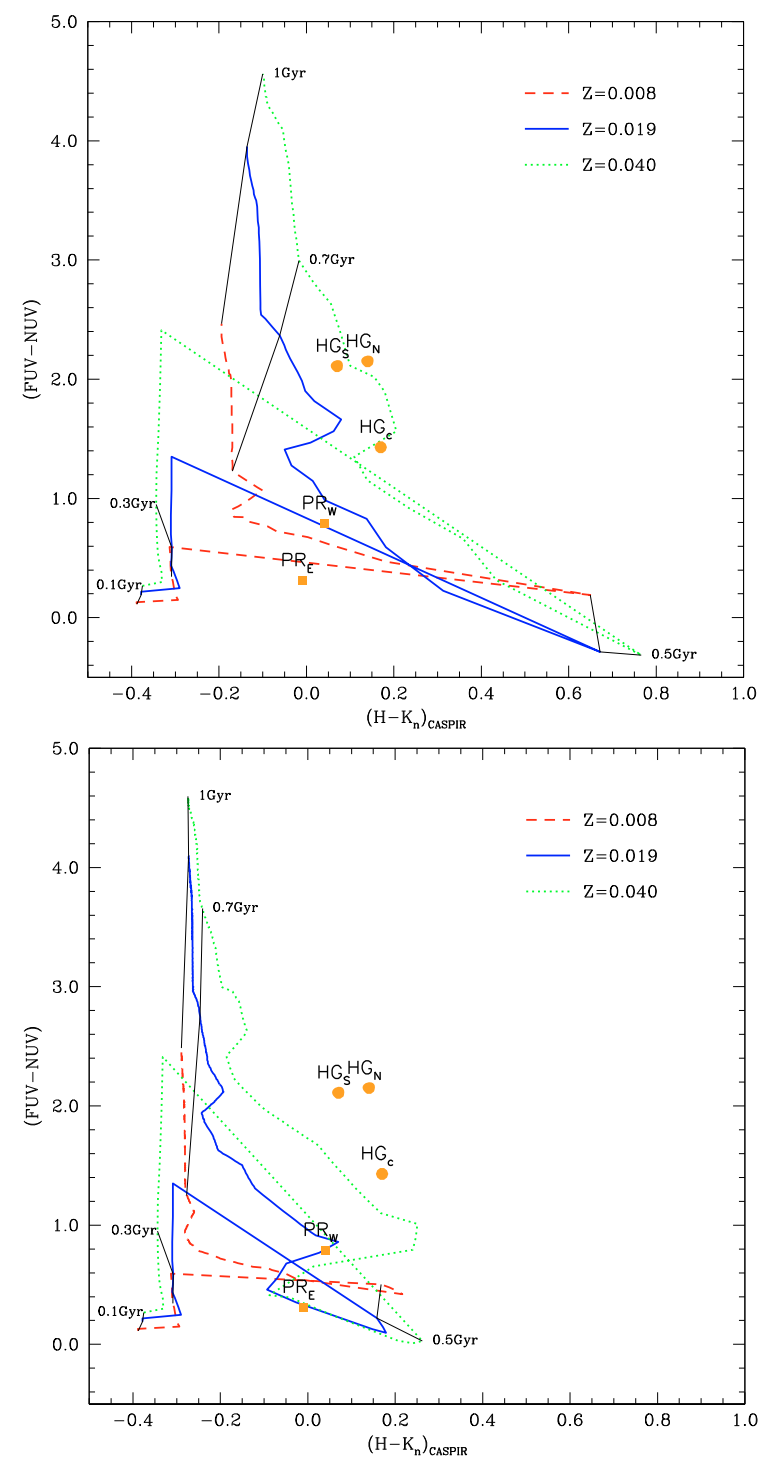

Fig. 13. Comparison between theoretical colour-colour relations and GALEX, optical, and NIR measurements. We plot the effect of a recent burst of star formation involving 70\% (upper panel) or 30\% (lower panel) of the stellar population. SSPs with self-contamination by dust are considered. The data for the eastern and western regions of the MCG-05-07-001 ring $\left(P R_{\mathrm{W}}\right.$ and $\left.P R_{\mathrm{E}}\right)$ and the northern, central, and southern regions of the host galaxy $\left(H G_{\mathrm{N}}, H G_{\mathrm{C}}, H G_{\mathrm{S}}\right)$ are shown. The colours of the ring and host galaxy are indicative the presence of a very young stellar population.

or a young metal-rich population. This is the well-known agemetallicity degeneracy, which cannot be broken in the colourcolour plane GALEX $(F U V-N U V)$ and $\left(H-K_{\text {CASPIR }}\right)$. The only conclusion that we can draw is that a strong contribution by a young obscured population seems to be needed to reproduce the red colours, but not much can be said about the underlying population on which the burst is superimposed.

\subsection{A detailed dusty EPS model for MCG-05-07-001}

The use of both dust-free and dusty SSPs (or SEDs), roughly mimicking a mixture of old (dust-free) and young (dust embedded) stellar populations in active galaxies, has provided general clues about the age and intensity of the recent burst of star formation. However, a galaxy is a complex object, whose star 
formation history cannot be reduced simply to the sum of two SSPs. For this reason, we extend the complexity of our analysis by using realistic evolutionary population synthesis (EPS) models of galaxies and apply this technique to interpret the data of MCG-05-07-001. For the aims of the present study, the EPS must include the contribution of dust to the extinction in the UV-optical range. Unfortunately, no data are available in the MIR/FIR range for MCG-05-07-001: these would help us to firmly constrain the dust content of the galaxy, the SED, and finally the star-formation history ( $\mathrm{SFH}$ ) of this galaxy.

The EPS models in use here are based on a robust model of chemical evolution that takes into account both dusty and dust-free SSPs, and include a realistic description of the geometrical shape of a galaxy in which stars and dust are distributed (Silva et al. 1998; Piovan et al. 2006b). The chemical model includes prescriptions for the infall of primordial gas, the initial mass function (IMF), the star-formation rate (SFR), and the stellar ejecta and provides the total amounts of gas and stars present at any age together with their chemical history (see Tantalo et al. 1996 , for more details). The chemical model provides the enrichment law $Z(t)$, the SFH, and the gas content to be supplied to the EPS model (with dust).

The model that we adopt to analyse MCG-05-07-001 takes into account the three different components of the dust: (i) the diffuse interstellar medium (ISM) composed of gas and dust; (ii) the star-forming regions such as the large complexes of MCs and, finally; (iii) stars of any age and chemical composition. The library of dusty SEDs of Piovan et al. (2006a), described in Sect. 5.1 is used to model young star-forming regions, whereas the dust-free SSPs are included for the stars already free from the parental MCs.

The dust description for the diffuse ISM is included according to the widely used silicates-graphite-PAH paradigm. The properties of dust follow Weingartner \& Draine (2001a) for the distribution of the grains, and Li \& Draine (2001) for the optical properties. To describe the evolution of the extinction curve with metallicity we use the sequence of models proposed by Weingartner \& Draine (2001a) that are able to reproduce the SMC/LMC/MW properties. Indeed, SMC, LMC and MW represent a sequence of models of increasing metallicity, which together with the different composition pattern is the origin of their peculiar average extinction law (Calzetti et al. 1994). For the MW metallicity in particular, we adopted $R_{v}=3.1$ for the diffuse ISM and $R_{v}=5.5$ for young dusty MCs. Also, eventually for $Z>Z_{\odot}$, the dust-to-gas ratio scales linearly according to $\delta=\delta_{\odot}\left(Z / Z_{\odot}\right)$ but for the same abundance pattern as the MW. The emission properties of graphite, silicates, and PAHs are computed according to Puget et al. (1985); Guhathakurta \& Draine (1989); Weingartner \& Draine (2001b); Draine \& Li (2001); Li \& Draine (2001) (see Piovan et al. 2006a,b, for a more detailed explanation).

The extinction in our model therefore works in two phases: (i) young stars are attenuated by the parental MC until the cloud is removed by $\mathrm{SN}$ explosions and stellar winds from high-mass stars; (ii) all the stars of any age and metallicity are attenuated by the diffuse ISM (see Silva et al. 1998; Charlot \& Fall 2000, for other examples of similar attenuation schemes).

Finally, to calculate the outgoing radiation we need to describe the interplay between stars and ISM in producing up the total SED. To this aim, it is necessary to know how the stars and ISM are distributed across a galaxy. The total mass of the gas and stars provided by the chemical model are then distributed over the entire galaxy volume by means of suitable density profiles that depend on the galaxy type (spheroidal, disc, and disc
Table 6. Relevant properties of the adopted chemical and dusty model for the simulated galaxy.

\begin{tabular}{ll}
\hline \hline \multicolumn{2}{c}{ The basic chemical model } \\
\hline Total mass of galaxy $M_{\text {tot }}$ & $1.30 \times 10^{10} M_{\odot}$ \\
Total mass of gas $M_{\text {gas }}$ & $7.82 \times 10^{9} M_{\odot}$ \\
Total mass of stars $M_{\text {tot }}$ & $5.06 \times 10^{9} M_{\odot}$ \\
Fraction of gas $M_{\text {gas }} / M_{\text {tot }}$ & 0.6 \\
Metallicity $Z$ & 0.0178 \\
Time of galactic wind ${ }^{a}$ & $1.09 \mathrm{Gyr}$ \\
\hline The associated EPS model with dust \\
Total radius & $15 \mathrm{Kpc}$ \\
Fraction of molecular gas $M_{\text {mol }} / M_{\text {gas }}$ & 0.3 \\
Evaporation time of $\mathrm{MCs}^{b}$ & $30 \mathrm{Myr}$ \\
\hline
\end{tabular}

${ }^{a}$ Duration of the SFH; ${ }^{b}$ time scale needed to evaporate the MC in which a new generation of stars is embedded.

plus bulge). To calculate the total SED, the galaxy model is divided into volume elements each of which is, at the same time, a source of radiation from the inner components inside and the absorber/emitter of radiation from and to all other volume elements (see Piovan et al. 2006a,b, for more details).

We adapt the above model to reproduce features of MCG05-07-001, which has a total mass $M_{\text {tot }} \sim 1.3 \times 10^{10} M_{\odot}$. From Table 1 , we know that the total mass of gas $\left(M_{\text {gas }}\right)$ is $\sim 8 \times 10^{9} M_{\odot}$, assuming that $M_{\mathrm{gas}}=M_{\mathrm{HI}}+M_{\mathrm{H}_{2}}$. The gas and total mass are taken as basic constraints on the chemical model best suited to represent MCG-05-07-001 (Tantalo et al. 1996), which is imposed to match the ratio $M_{\text {gas }} / M_{\text {tot }} \sim 0.6$.

The key properties of this model are listed in the top part of Table 6, whereas the bottom part lists the key parameters adopted to simulate the effect of dust (see Piovan et al. 2006b, for all details). Although MCG-05-07-001 has a complex geometrical structure, for the purposes of a qualitative analysis, and to distribute gas (dust) and stars across the galaxy, we can adopt the King profile (Piovan et al. 2006b). Furthermore, the relative proportion of gas in the diffuse ISM and cold MCs given in Table 1 is implied by the observational data, as for the galaxy radius. This is derived from the observational data and set to be equal to two times the effective radius $r_{\mathrm{e}}$, and is about $7.8 \mathrm{kpc}$ according to Whitmore et al. (1987). The MC evaporation time is chosen according to the average lifetime of the most massive stars, on the notion that this is roughly the timescale required by stars to expel their parental cloud.

For the three selected areas across the galaxy for which we have measurements of the flux (magnitudes) in six different photometric bands from the far UV to the near IR ( $F U V, N U V, B, J, H, K n$ ), we compare the observational magnitudes with the theoretical counterparts derived from the chemospectro-photometric models of the galaxy presented above. To this aim, an important quantity to know is the fraction of galaxy mass encompassed by the aperture in usage. To estimate the mass, we calculate how much galaxy light falls within the aperture located at the two sides of the ring and the main body of the galaxy, which is approximated to be an oblate disk. We then calculate the amount of stellar light integrated along the lineof-sight using the formalism developed by Galletta (1984). In brief, we assume: 1) an oblate disk with intrinsic axial ratio 0.25 ; 2) an exponential intrinsic density profile, with the same effective radius of the MCG-05-07-001 luminosity profile (19"); 3) a 


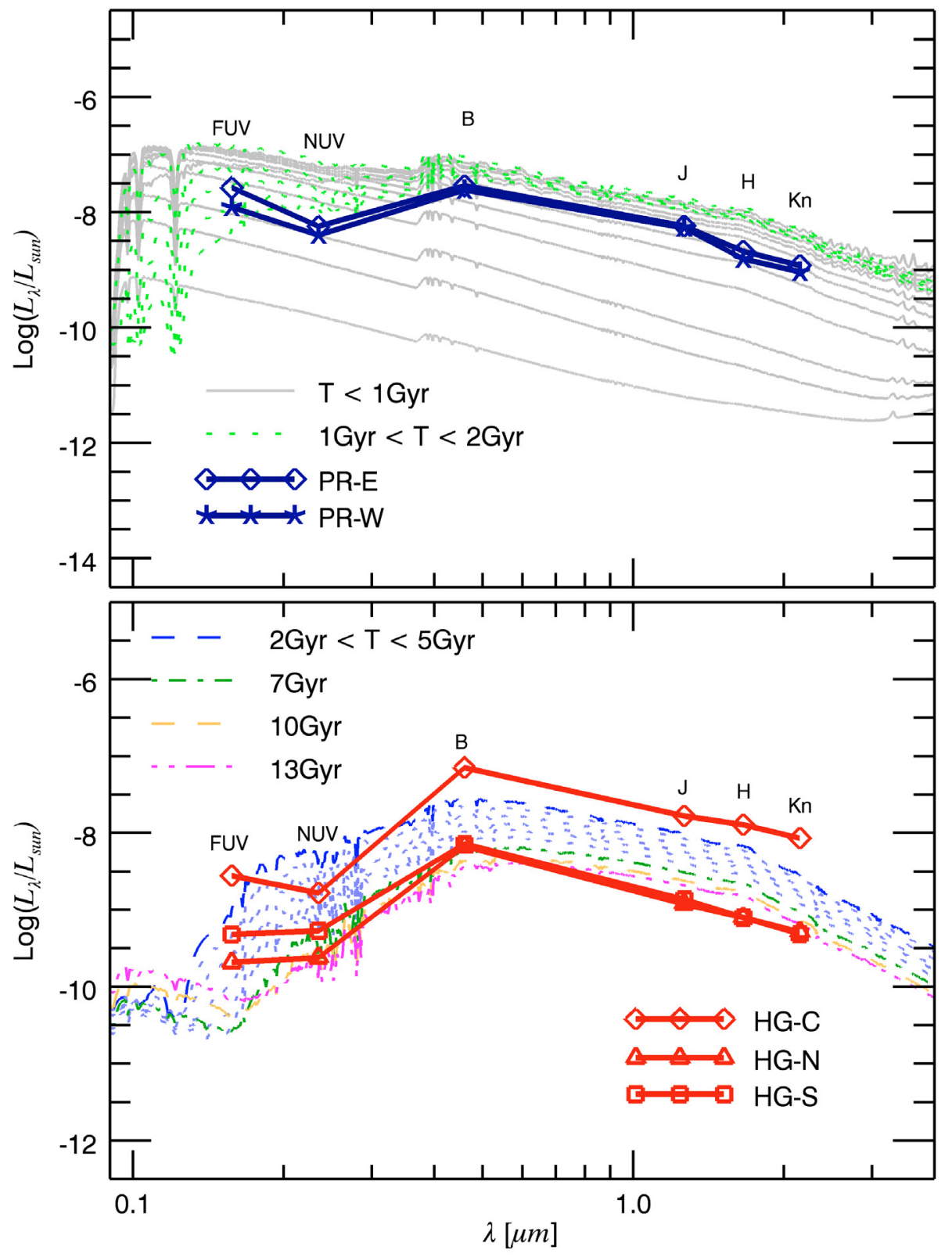

Fig. 14. Dusty EPS galaxy models of different ages are superimposed on the SED of MCG05-07-001 for which six observational measurements from the far-UV to the near IR are available. Top panel: comparison between the theoretical spectra up to the age of $2 \mathrm{Gyr}$ and the aperture magnitudes of the east and west sides of the polar ring. Bottom panel: comparison between the theoretical spectra in the range 2-13 Gyr and the aperture magnitudes derived on the northern, central and southern regions of the body of the host galaxy along the major axis (see the text for details). constant $\mathrm{M} / \mathrm{L}$ ratio across the galaxy; 4) a total galaxy extension of $\left.2.35^{\prime} ; 5\right)$ that the whole galaxy can be described by a model made of 100 shells with same intrinsic flattening. From the apparent axial ratio of 0.35 , we calculate the inclination of the line-of-sight with respect to the galaxy plane, which amounts to $75.4^{\circ}$. The circular apertures are approximated by summing up 13 line-of-sights, each of which are $1 \times 1$ pixel in area $(1.5 \times 1.5$ " in our case). A correction has been added to the integrated values, to take into account the difference between the area covered by the 13 pixels and that of a perfect circle. The final result indicates that the aperture pointed toward the main body contains $\sim 3 \%$ of its total mass (excluding the ring), whereas the apertures pointed toward the wings each include $0.7 \%$ of the total mass. This result is not influenced by the assumption about the disk flattening, as the galaxy is seen nearly edge-on. The data plotted in Fig. 14 are scaled by taking into account these percentages.

As far as the corresponding theoretical magnitudes are concerned, we estimate the flux falling into each aperture in the following way. We start from the definition of $A B$ mag, properly rescale for the expected flux in the aperture, and finally calculate the luminosity in the passband to be compared with the observational data. The theoretical and observational results are compared in Fig. 14, where the theoretical luminosities (in magnitude scale) are shown for different values of the age of the EPS model.

The top panel refers to the data for the two windows that we placed on the two sides of the MCG-05-07-001 polar ring (labelled PR in Table 5). The bottom panel shows the same but for the windows that we placed on the main body of the host galaxy (labelled HG in Table 5). In this case, the observational sources are embedded in the HI gas as shown in Fig. 7. Finally, in Fig. 15 we show the difference between the theoretical and the observational luminosities. The legend has the same meaning as in Fig. 14. The age derived for the best-fits is also indicated.

The trend of the FUV and NUV fluxes in Fig. 14 for the PR data suggests the presence of a young stellar population $(\leq 1 \mathrm{Gyr})$, whereas the data for the remaining passbands from $B$ to $K_{n}$ seem to indicate the presence of a 2 Gyr population. Using 

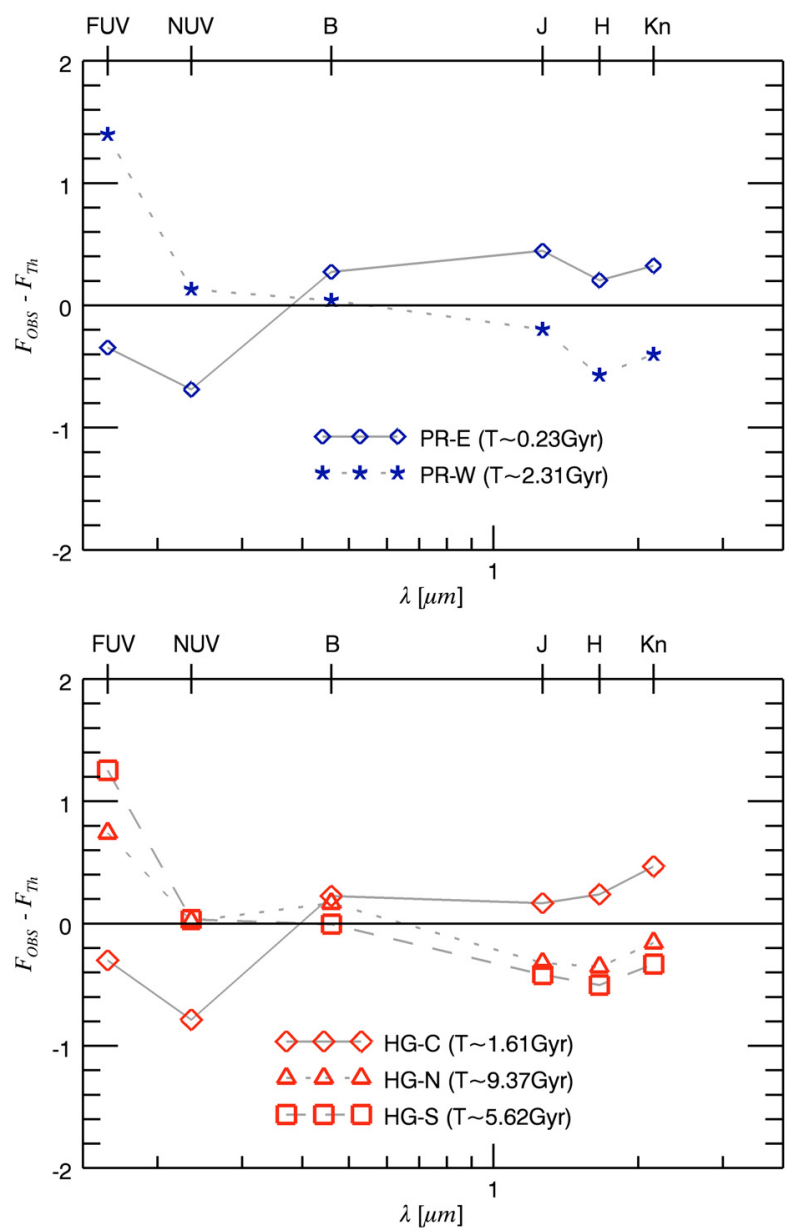

Fig. 15. Residuals between the dusty EPS galaxy models and observational data for the six observational data available for MCG-05-07-001, from the far-UV to the near IR. Only the best-fit models are presented together with the derived age. Top panel: residuals between the theoretical SED and the aperture magnitudes of the eastern $(P R$-E) and western part $(P E-\mathrm{W})$ of the polar ring. Bottom panel: residuals between the theoretical SED and the aperture magnitudes of the northern $(H G-\mathrm{N})$, central $(H G-\mathrm{C})$, and southern $(H G-\mathrm{S})$ regions of the body of the host galaxy along the major axis.

a best-fit procedure, are found the ages to be $0.23 \mathrm{Gyr}$ and 2.31 Gyr in the spectral ranges, FUV and NUV, and from $B$ to $K_{n}$, respectively, for both PR data. On the other hand, the three SEDs obtained with apertures located on the HG are more accurately described by EPS models with ages in the range 2-9 Gyr. The best-fit to the three HG regions are consistent with this age interval, as shown in Fig. 15.

Despite all of these uncertainties, these results are consistent with those obtained in Sect. 5.1 using simple SSPs: the MCG05-07-001 colours can be reproduced by assuming a recent, active burst of star formation occurred in the PR superimposed on an older population of stars in the HG. The analysis carried out with SEDs seems to favour this possibility, already proposed in Sect. 5.1, with respect to the one presented in Fig. 13, where both the burst and the underlying population were young. Our analysis therefore suggests that a very recent burst of star formation has been triggered probably by a gas rich wet accretion event onto an underlying older stellar system.

Finally, it is worth noting that even the best-fit to the SEDs does not give fully satisfactory results for all passbands and that the uncertainties are still large. We tried to vary some basic parameters of the model, such as the evaporation timescale of parental MCs, without substantially improving the results. The adopted geometry is probably too simple for a very irregular and complex system such as MCG-05-07-001, for which a proper treatment would require a more complicated description of the spatial distribution of dust and stellar sources.

\section{Summary and conclusions}

Systems of shells and polar rings in early-type galaxies are considered to be tracers of accretion and merger events. Their high frequency in low density environments suggests that these episodes could drive the secular evolution of at least a fraction of the early-type galaxy population.

We have obtained the surface photometry of three early-type galaxies observed with GALEX: MCG-05-07-001, a well-known polar-ring galaxy, and NGC 1210 and NGC 5329, which both display shell systems. In the present study, only NGC 5329 belongs to a poor galaxy structure (WBL 472), the others being very isolated galaxies. NGC 1210 and MCG-05-07-001 have a large quantity of HI gas associated with their stellar body.

Both the GALEX NUV and FUV images of MCG-05-07-001 and NGC 1210 detect complex tidal tails and debris structures. In the FUV, MCG-05-07-001 and NGC 1210 may be hardly classified as "classical" early-type galaxies from their morphology. In NGC 1210, inner shells are visible in the NUV image. The inner shell (labelled with " 2 " in Fig. 3) probably has a different origin from the outer ones, as suggested by the tail-like structure that it has in the FUV image. A case of multiple accretion has also been suggested for the shell galaxy NGC 474 by Rampazzo et al. (2006) and Sikkema et al. (2007).

The polar ring of MCG-05-07-001 is clearly visible in data for both GALEX bands, while the host galaxy, from which the bulk of the emission in optical and near infrared originates is almost undetected. GALEX data of NGC 5329 show that the distribution/morphology of its NUV emission is comparable to that of the optical image, while FUV emission is evident only in the central regions of the galaxy. This implies that the NUV emission originates in the same kind of stellar population. The FUV emission, which is more concentrated toward the nucleus, suggests that different type of hot stars contribute to the flux (see also Rampazzo et al. 2007). NGC 5329 does not show evidence of shells in the GALEX bands.

We used optical and near-infrared images to build coherent pictures of stellar content in both the nuclear region and the outskirts of the galaxies as well as the region where fine structures are present. We investigate the capability of the combined far UV, optical, and NIR colours to provide information about the time at which the accretion/merging phenomenon occurred. Our models suggest that a very young $(\leq 1 \mathrm{Gyr})$ stellar population is present in MCG-05-07-001: we cannot exclude the possibility of ongoing star formation in this galaxy. The nuclei of NGC 1210 and NGC 5329 also appear rejuvenated but the accretion episode that triggered these star formation episodes should be much older (2-4 Gyr).

Furthermore, the age estimated for the polar ring is consistent with the stability timescales of the polar structure formed by the accretion scenario: a low inclined polar ring (i.e., nearly polar) may be observed after several Gyr (Bournaud \& Combes 2003).

Van Dokkum (2005) suggested that dry mergers, i.e. nearly dissipation-less or gas less mergers at low redshift, are responsible for much of the local bright field elliptical galaxy population. Oddly enough, about $50 \%$ of nearby early-type galaxies 
were found to contain detectable HI (see e.g., Schiminovich 2001; Serra et al. 2007). MCG-05-07-001 and NGC 1210 are examples of wet mergers: they are dynamically young objects according to their irregular HI gas spatial distribution and young stellar population. GALEX images display unambiguously the strong morphological connection between the far UV and the HI distribution shedding light on the secular evolution of early-type galaxies and the final fate of the accreted gas. The presence of a young stellar population is then connected to the recent accretion and the refuelling of fresh gas to an otherwise old galaxy, rejuvenating its star formation.

Acknowledgements. We acknowledge partial financial support of the Agenzia Spaziale Italiana under contract ASI-INAF contract I/023/05/0. A.M. acknowledges partial financial support from The Italian Scientists and Scholars in North America Foundation (ISSNAF) and J. Herald for help in revising the text. GALEX is a NASA Small Explorer, launched in April 2003. GALEX is operated for NASA by California Institute of Technology under NASA contract NAS-98034. This research has made use of the SAOImage DS9, developed by Smithsonian Astrophysical Observatory and of the NASA/IPAC Extragalactic Database (NED) which is operated by the Jet Propulsion Laboratory, California Institute of Technology, under contract with the National Aeronautics and Space Administration. IRAF is distributed by the National Optical Astronomy Observatories, which are operated by the Association of Universities for Research in Astronomy, Inc., under cooperative agreement with the National Science Foundation. We acknowledge the usage of the HyperLeda database (http://leda.univ-lyon1.fr). The Digitized Sky Survey (DSS) was produced at the Space Telescope Science Institute under US Government grant NAG W-2166. The images of these surveys are based on photographic data obtained using the Oschin Schmidt Telescope at the Palomar Observatory and the UK Schmidt Telescope. The plates were processed into the present compressed digital form with the permission of these institutions.

\section{References}

Aarseth, S. J., \& Fall, S. M. 1980, ApJ, 236, 43

Balcells, M., van Gorkom, J. H., Sancisi, R., et al. 2001, AJ, 122, 1758

Barnes, J. 1985, MNRAS, 215, 517

Barnes, J. E. 1992, ApJ, 393, 484

Bekki, K. 1998, ApJ, 499, 635

Bournaud, F., \& Combes, F. 2003, A\&A, 401, 817

Bournaud, F., Jog, C. J., \& Combes, F. 2005, A\&A, 437, 69

Bressan, A., Chiosi, C., \& Fagotto, F. 1994, ApJS, 94, 63

Bressan, A., Granato, G. L., \& Silva, L. 1998, A\&A, 332, 135

Brocca, C., Bettoni, D., \& Galletta, G. 1997, A\&A, 326, 907

Brook, C. B., Governato, F., Quinn, T., et al. 2009, ApJ, 694, 396

Brown, R. J. N., Forbes, D. A., Silva, D., et al. 2003, MNRAS, 341, 747

Calzetti, D., Kinney, A. L., \& Storchi-Bergmann, T. 1994, ApJ, 429, 582

Charlot, S., \& Fall, S. M. 2000, ApJ, 539, 718

Charmandaris, V., Combes, F., \& van der Hulst, J. M. 2000, A\&A, 356, L1

Christodoulou, D. M., Katz, N., Rix, H.-W., et al. 1992, ApJ, 395, 113

Colbert, J. W., Mulchaey, J. S., \& Zabludoff, A. I. 2001, AJ, 121, 808

Davé, R., Cen, R., Ostriker, J. P., et al. 2001, ApJ, 552, 473

Draine, B. T. 2003, ARA\&A, 41, 241

Draine, B. T., \& Li, A. 2001, ApJ, 551, 807

Dupraz, C., \& Combes, F. 1986, A\&A, 166, 53

Ebeling, H., White, D. A., \& Rangarajan, F. V. N. 2006, MNRAS, 368, 65

Fabian, A. C., Nulsen, P. E. J., \& Stewart, G. C. 1980, Nature, 287, 613

Galletta, G. 1984, Ap\&SS, 103, 39

Galletta, G., Sage, L. J., \& Sparke, L. S. 1997, MNRAS, 284, 773

Guhathakurta, P., \& Draine, B. T. 1989, ApJ, 345, 230

Hernquist, L., \& Mihos, J. C. 1995, ApJ, 448, 41

Hernquist, L., \& Quinn, P. J. 1987a, ApJ, 312, 17

Hernquist, L., \& Quinn, P. J. 1987b, ApJ, 312, 1

Hernquist, L., \& Spergel, D. N. 1992, ApJ, 399, L117

Hibbard, J. E., Bianchi, L., Thilker, D. A., et al. 2005, ApJ, 619, L87

Iodice, E., Arnaboldi, M., Sparke, L. S., et al. 2002a, A\&A, 391, 117
Iodice, E., Arnaboldi, M., Sparke, L. S., Gallagher, J. S., \& Freeman, K. C. 2002b, A\&A, 391, 103

Jedrzejewski, R. I. 1987, MNRAS, 226, 747

Katz, N., \& Rix, H.-W. 1992, ApJ, 389, L55

Li, A., \& Draine, B. T. 2001, ApJ, 554, 778

Longhetti, M., Rampazzo, R., Bressan, A., et al. 1998a, A\&AS, 130, 251

Longhetti, M., Rampazzo, R., Bressan, A., et al. 1998b, A\&AS, 130, 267

Longhetti, M., Bressan, A., Chiosi, C., et al. 1999, A\&A, 345, 419

Longhetti, M., Bressan, A., Chiosi, C., et al. 2000, A\&A, 353, 917

Macciò, A. V., Moore, B., \& Stadel, J. 2006, ApJ, 636, L25

Malin, D. F., \& Carter, D. 1983, ApJ, 274, 534

Marigo, P., Girardi, L., Bressan, A., et al. 2008, A\&A, 482, 883

Martin, D. C., Fanson, J., Schiminovich, D., et al. 2005, ApJ, 619, L1

Merritt, D. 1985, ApJ, 289, 18

Morrissey, P., Schiminovich, D., Barlow, T. A., et al. 2005, ApJ, 619, L7

Morrissey, P., Conrow, T., Barlow, T. A., et al. 2007, ApJS, 173, 682

Peng, C. Y., Ho, L. C., Impey, C. D., et al. 2002, AJ, 124, 266

Pierfederici, F., \& Rampazzo, R. 2004, Astron. Nach., 325, 359

Piovan, L., Tantalo, R., \& Chiosi, C. 2003, A\&A, 408, 559

Piovan, L., Tantalo, R., \& Chiosi, C. 2006a, MNRAS, 366, 923

Piovan, L., Tantalo, R., \& Chiosi, C. 2006b, MNRAS, 370, 1454

Prieur, J.-L. 1990, Status of shell galaxies, ed. R. Wielen, 72

Puget, J. L., Leger, A., \& Boulanger, F. 1985, A\&A, 142, L19

Rampazzo, R., Plana, H., Longhetti, M., et al. 2003, MNRAS, 343, 819

Rampazzo, R., Alexander, P., Carignan, C., et al. 2006, MNRAS, 368, 851

Rampazzo, R., Marino, A., Tantalo, R., et al. 2007, MNRAS, 381, 245

Reduzzi, L., Longhetti, M., \& Rampazzo, R. 1996, MNRAS, 282, 149

Reshetnikov, V., \& Sotnikova, N. 1997, A\&A, 325, 933

Salim, S., Rich, R. M., Charlot, S., et al. 2007, ApJS, 173, 267

Schiminovich, D. 2001, in Gas and Galaxy Evolution, ed. J. E. Hibbard,

M. Rupen, \& J. H. van Gorkom, ASP Conf. Ser., 240, 147

Schiminovich, D., van Gorkom, J. H., van der Hulst, J. M., et al. 1994, ApJ, 423, L101

Schiminovich, D., van Gorkom, J. H., van der Hulst, J. M., et al. 1995, ApJ, 444, L77

Schiminovich, D., van Gorkom, J., van der Hulst, T., Oosterloo, T., \& Wilkinson, A. 1997, in The Nature of Elliptical Galaxies; 2nd Stromlo Symposium, ed. M. Arnaboldi, G. S. Da Costa, \& P. Saha, ASP Conf. Ser., 116, 362

Schiminovich, D., van Gorkom, J. H., Dijkstra, M., et al. 2001, in Gas and Galaxy Evolution, ed. J. E. Hibbard, M. Rupen, \& J. H. van Gorkom, ASP Conf. Ser., 240, 864

Schweizer, F. 1993, in Structure, Dynamics and Chemical Evolution of Elliptical Galaxies, ed. P. Poulain, \& E. Davoust, 651

Schweizer, F., \& Seitzer, P. 1992, AJ, 104, 1039

Semelin, B., \& Combes, F. 2005, A\&A, 441, 55

Serra, P., \& Trager, S. C. 2007a, MNRAS, 374, 769

Serra, P., Trager, S. C., van der Hulst, J. M., et al. 2007, New Astron. Rev., 51, 3

Siebenmorgen, R., \& Krügel, E. 2007, A\&A, 461, 445

Sikkema, G., Carter, D., Peletier, R. F., et al. 2007, A\&A, 467, 1011

Silva, L., Granato, G. L., Bressan, A., et al. 1998, ApJ, 509, 103

Stoughton, C., Lupton, R. H., Bernardi, M., et al. 2002, AJ, 123, 485

Takagi, T., Vansevicius, V., \& Arimoto, N. 2003, PASJ, 55, 385

Tantalo, R., Chiosi, C., Bressan, A., et al. 1996, A\&A, 311, 361

Thakar, A. R., \& Ryden, B. S. 1996, ApJ, 461, 55

Thakar, A. R., \& Ryden, B. S. 1998, ApJ, 506, 93

Theureau, G., Hanski, M. O., Coudreau, N., Hallet, N., \& Martin, J.-M. 2007, A\&A, 465, 71

Thomson, R. C. 1991, MNRAS, 253, 256

Thomson, R. C., \& Wright, A. E. 1990, MNRAS, 247, 122

Van Dokkum, P. G. 2005, AJ, 130, 2647

van Gorkom, J., \& Schiminovich, D. 1997, in The Nature of Elliptical Galaxies;

2nd Stromlo Symposium, ed. M. Arnaboldi, G. S. Da Costa, \& P. Saha, ASP Conf. Ser., 116, 310

van Gorkom, J. H., Schechter, P. L., \& Kristian, J. 1987, ApJ, 314, 457

Weil, M. L., \& Hernquist, L. 1993, ApJ, 405, 142

Weingartner, J. C., \& Draine, B. T. 2001a, ApJ, 548, 296

Weingartner, J. C., \& Draine, B. T. 2001b, ApJ, 563, 842

White, R. A., Bliton, M., Bhavsar, S. P., et al. 1999, AJ, 118, 2014

Whitmore, B. C., McElroy, D. B., \& Schweizer, F. 1987, ApJ, 314, 439

Whitmore, B. C., Lucas, R. A., McElroy, D. B., et al. 1990, AJ, 100, 1489

Wilkinson, A., Prieur, J.-L., Lemoine, R., et al. 2000, MNRAS, 319, 977

Williams, R. E., \& Christiansen, W. A. 1985, ApJ, 291, 80 